\title{
Bedeutung des Geschiebetransportes für die Planung von Hochwasserschutz- und Retentionsmaßnahmen in Talflüssen - Numerische Modellierung des Inns im Tiroler Unterinntal
}

\author{
Katharina Baumgartner · Bernhard Gems · Stefan Walder · Florian Auer · Markus Federspiel · Markus Aufleger \\ Online publiziert: 6. März 2017 \\ (c) Der/die Autor(en) 2017. Dieser Artikel ist eine Open-Access-Publikation.
}

Zusammenfassung Die Bedeutung des Hochwasserrisikomanagements im Alpenraum nimmt immer stärker zu. Ziel der Planung ist die Verringerung der hochwasserbedingten nachteiligen Folgen auf die menschliche Gesundheit, die Umwelt, das Kulturerbe und wirtschaftliche Tätigkeiten. Der Inn im Tiroler Unterinntal steht gegenwärtig im Mittelpunkt intensiver Untersuchungen zur Verbesserung des Hochwasserschutzes der angrenzenden Siedlungsräume. Die Betrachtung des Feststoffhaushaltes eines Fließgewässers bzw. die sich im Verlauf eines Hochwassers einstellenden Sohllagenänderungen können einen maßgeblichen Einfluss auf die Wirkung von Hochwasserschutzmaßnahmen und die Dotierung von Retentionsräumen haben. Der vorliegende Artikel befasst sich mit der Bedeutung des Geschiebetransportes für die schutzwasserbaulichen Planungen am Inn, welche Maßnahmen zum Schutz von Siedlungs- und Gewerbegebieten, wie Linearmaßnahmen, Erhalt der natürlichen

DI K. Baumgartner $(\bowtie)$.

DI Dr. B. Gems .

Univ.-Prof. Dr.-Ing. M. Aufleger Arbeitsbereich Wasserbau Institut für Infrastruktur, Universität Innsbruck, Technikerstraße 13, 6020 Innsbruck, Österreich

katharina.baumgartner@uibk.ac.at

DI Dr. S. Walder · DI M. Federspiel Abteilung Wasserwirtschaft Sachgebiet Schutzwasserwirtschaft und Gewässerökologie, Amt der Tiroler Landesregierung, Herrengasse 1-3, 6020 Innsbruck, Österreich

DI F. Auer

ATP architekten ingenieure,

Heiliggeiststraße 16, 6010 Innsbruck, Österreich
Überflutungsräume und ungesteuerte und über ein Pegelkollektiv gesteuerte Retentionsräume umfasst. Anhand einer fraktionierten Geschiebetransportberechnung für den $73,5 \mathrm{~km}$ langen Gewässerabschnitt konnten sensible Bereiche für die Dotierung der Retentionsräume ausgemacht werden. Der Einfluss der morphologischen Änderungen bzw. der sich einstellenden Sohllagenänderungen während eines 100-jährlichen Hochwasserereignisses auf die Planung der Hochwasserschutzund Retentionsmaßnahmen konnte aufgezeigt werden. Die aus dem durchgeführten Projekt gewonnenen Modellierungsergebnisse bilden eine wichtige Grundlage für die weiteren Planungsschritte, um eine hinreichende und optimale Scheitelreduktion im Hochwasserfall sicher zu stellen.

Significance of bed-load transport for flood protection and retention measures in alpine rivers - Numerical modeling of the Inn River in the Lower Inn Valley

Abstract Flood risk management has become increasingly important in Alpine regions. Hence, the Inn River located in the lower Inn valley is focus of intensive research that aims to decrease flood risks for the densely settled valley plain. The movement of sediments within the river system or rather the development of the river bed during floods can have a significant impact on the effectiveness of flood protection measures and the dimensioning of retention areas. The study presented in this paper deals with flood mitigation systems for the Inn River, such as measures for protection of individual objects, lateral protection structures and uncontrolled and controlled retention areas. Through the project, under- standing of potential impacts of morphodynamic changes on the efficiency and functionality of flood-protection measures was achieved. In order to identify sensitive areas for retention control, a multi-fractioned bed-load transport simulation was applied for the $73,5 \mathrm{~km}$ long reach of the Inn River. The simulation could prove the influence of morphological changes and resulting river bed formations during a $100 \mathrm{yr}$-flood on flood protection and retention measures. Presented modelling results aim to provide a profound basis for the further planning procedure of flood protection and retention measures in order to reach an efficient and optimized peak reduction in case of floods.

\section{Einleitung}

Schutz von Siedlungsräumen vor Hochwässern, die Erhaltung und Wiederherstellung ökologisch wertvoller Gewässerstrukturen und die demografische Entwicklung prägen aktuell viele flussbauliche Projekte an alpinen Gewässern.

Flussbegradigungen, Laufverlegungen oder Regulierungen waren in der Historie allerorts umgesetzte flussbauliche Konzepte im Alpenraum. Damit konnten der Siedlungsraum und landwirtschaftliche Nutzflächen in den Einflussbereich der Gewässer erweitert werden. Die gegenwärtige Struktur des alpinen Dauersiedlungsraumes mit teilweise intensivster Nutzung der Talräume und der Schwemmkegelflächen resultiert aus dieser seit nunmehr vielen Jahren verfolgten Vorgehensweise. Damit steigt in diesen Gebieten das Schadenspotenzial. Natürliche Retentionsräume oder ökologisch wertvolle Vorländer stehen den Gewässern oft nur mehr in sehr geringem Umfang zur Verfügung (Bätzing 1991; Werth 2003). 



\section{Raum Hall in Tirol, 1946 - 2013 \\ Siedlungsentwicklung Unterinntal}

Raum Wörgl, 1973 - 2013
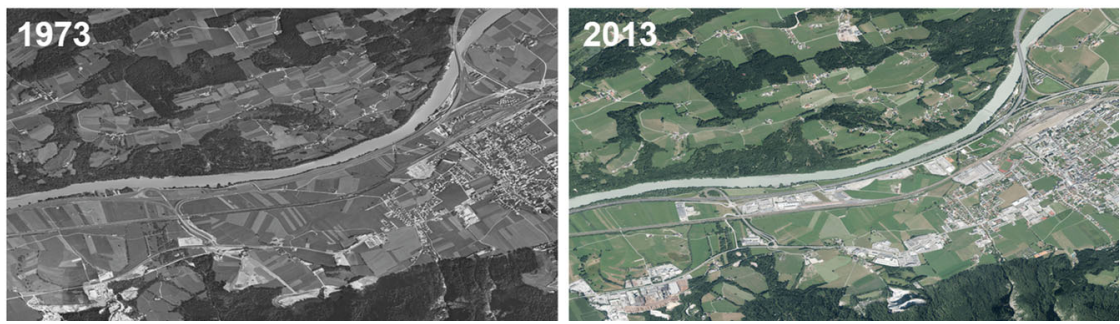

Abb. 1 Beispiele für die Siedlungsentwicklung im Inntal im Zeitraum 1946 bis 2013 (Daten: @ Land Tirol)

Abb. 1 zeigt in diesem Kontext die demografische Entwicklung im Inntal, beispielhaft für die Siedlungsräume um Hall in Tirol (Zeitraum 1946 bis 2013) und Wörgl (Zeitraum 1973 bis 2013). Die Ausdehnung bebauter Flächen in unmittelbarer Nähe zum Inn und den Zubringergewässern, sowohl für Wohnbau, gewerbliche Nutzung und auch für Infrastrukturobjekte, ist hier evident.

Aus schutzwasserbaulicher Sicht sind Maßnahmen der Kapazitätserhöhung alpiner Gewässer nach wie vor notwendig und sinnvoll. Im Rahmen integraler, nachhaltiger und ökologisch verträglicher Planungsansätze werden sie mittlerweile gemeinsam mit anderen flussbaulichen und raumplanerischen Maßnahmen, beispielsweise der Schaffung ökologischer Ausgleichsflächen oder der Freihaltung von Flächen für die Hochwasserretention, betrachtet (Aufleger et al. 2012; Geilen et al. 2004; Poulard et al. 2010; Zarn 2008).

Schadenbringende Hochwässer zeigen immer wieder auf, dass fluviatilen Gefahrenprozessen ausschließlich durch technische Schutzmaßnahmen nicht vollständig entgegnet werden kann. Extrem seltene Ereignisse, welche die Kapazitäten der Gewässer bzw. der Schutzbauwerke überschreiten, stellen ein gewisses Restrisiko für den angrenzenden Siedlungsraum dar. Diese Ereignisse können dort aufgrund des hohen Schadenspotenzials enorme Schäden verursachen (vgl. Abb. 1). Größere Hochwasserereignisse ziehen häufig auch eine Harmonisierung des Datenkollektivs zur Berechnung von hydrologischen Erwartungswerten mit definierten Jährlichkeiten nach sich.
Möglicherweise ergeben sich hieraus höhere Bemessungsabflüsse, welche der Dimensionierung von Hochwasserschutzmaßnahmen zugrunde gelegt werden. Hierdurch kann die Realisierung neuer bzw. auch die Anpassung bestehender Schutzmaßnahmen notwendig werden.

Für die Planung von Hochwasserschutzmaßnahmen sind zwei wesentliche Aspekte zu nennen:

1. Für Siedlungen und bedeutende Wirtschafts- und Verkehrsanlagen (höherwertige Nutzungen) ist grundsätzlich die Gewährleistung eines Schutzes bis $\mathrm{zu}$ Hochwasserereignissen mit einem voraussichtlichen Wiederkehrintervall von 100 Jahren $\left(\mathrm{HQ}_{100}\right)$ anzustreben.

2. Schutzmaßnahmen entlang des Gewässers sind so zu gestalten, dass sie im Vergleich zur Bestandssituation keine nachteiligen Konsequenzen für die Unterlieger verursachen. Es gilt in diesem Kontext das sogenannte „Verschlechterungsverbot“.

Aus beiden genannten Aspekten ergibt sich letztlich die Voraussetzung, dass eine Umsetzung von linearen Hochwasserschutzmaßnahmen zur Reduzierung von Überflutungen in besonders hochwertigen Räumen (z. B. Siedlungen, Gewerbegebiete) in Kombination mit Retentionsmaßnahmen erfolgen muss, welche dem Verschlechterungsverbot für die Unterlieger gerecht werden. Die Planung technischer Schutzmaßnahmen basiert entsprechend auf der Strecken- bzw. Einzugsgebietsebene (Habersack et al. 2011). Eine hinrei- chende Retentionswirkung entlang von Flüssen in vergleichsweise dicht bebauten Räumen ist vorwiegend mit einer Anordnung im Nebenschluss, d. h. durch Aktivierung bzw. Optimierung von Retentionsräumen, zu erreichen. Für eine effiziente Ausnutzung des Retentionsraumes ist die Steuerung der Zuflüsse in den Retentionsraum von wesentlicher Bedeutung (Fischer 2008). Der Betrieb der Einlaufbauwerke mit entsprechenden Verschlussorganen richtet sich dabei aus Gründen der Erhöhung der Zuverlässigkeit häufig nach den Abflüssen bzw. Wasserständen an mehreren Steuerpegeln im Gewässer. Nachdem letztlich nicht nur die Abflussspitze, sondern auch die Wellenform eines Hochwassers für eine optimale Ausnutzung von Retentionsräumen relevant ist, ist grundsätzlich insbesondere die Miteinbeziehung von Hochwasserprognosemodellen für das Einzugsgebiet des Gewässers eine wichtige Voraussetzung für eine möglichst effiziente Ausnutzung von Retentionsräumen (Fischer 2008; Homagk 2007). Hochwasserprognosemodelle sind aber nicht bei allen Gewässern (z. B. bei zu geringen Vorlaufzeiten) sinnvoll anzuwenden und ist daher im Einzelfall zu untersuchen.

Der Transport von Geschiebe im Gewässer bzw. die sich im Verlauf eines Hochwassers einstellenden Sohllagenänderungen können einen wesentlichen Einfluss auf die Wirkung von Hochwasserschutzmaßnahmen (Gems 2012; Plörer 2012) und die Dotierung von Retentionsräumen haben. Anlandungsprozesse beispielsweise aufgrund massiver Seiteneinträge ins Gewässer 

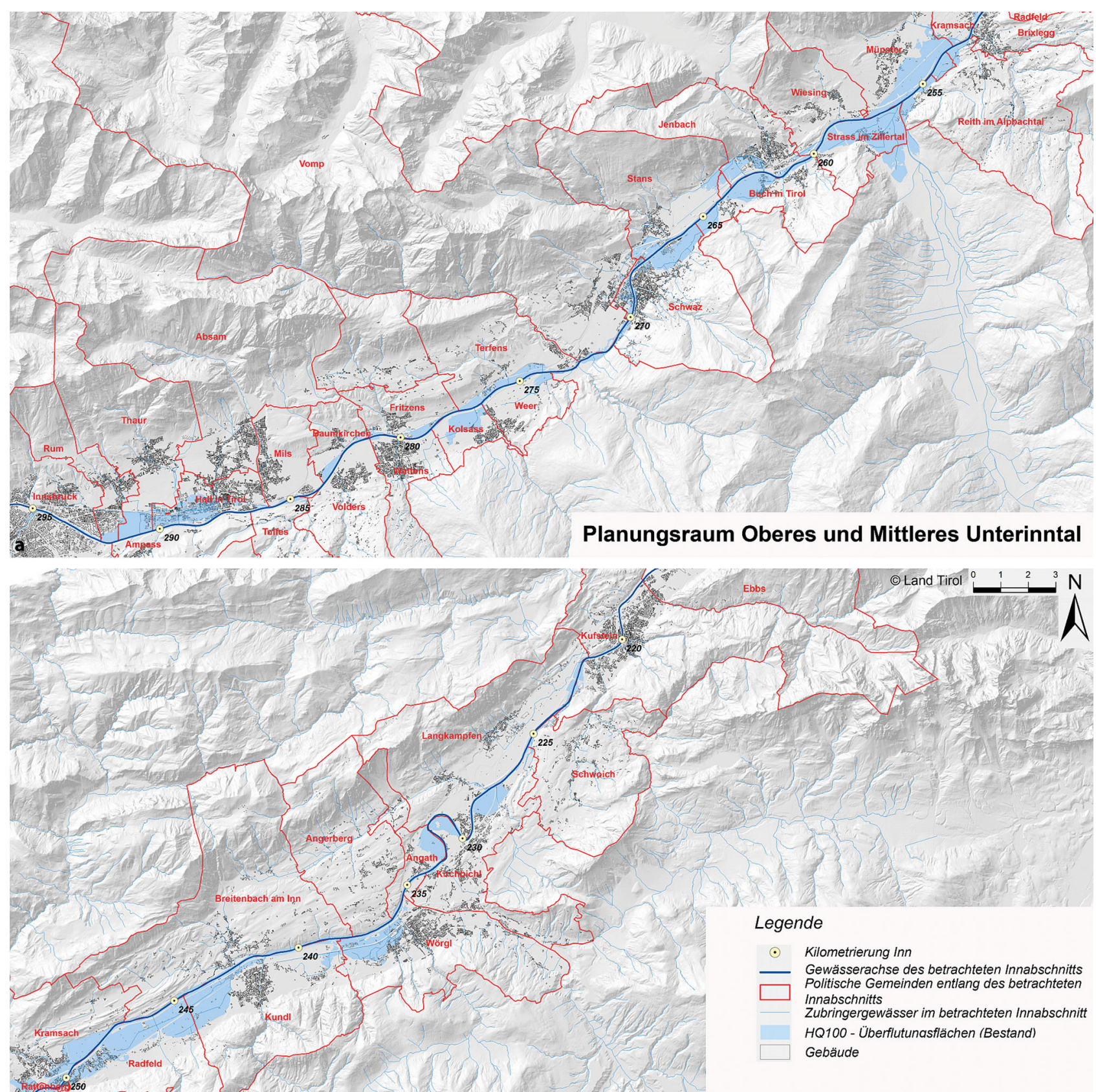

Abb. 2 Übersicht über das Projektgebiet des Inns im Unterinntal-Gewässerabschnitt von der Mündung der Sill in Innsbruck (km 294,7) bis zur Mündung der Weißache in Kufstein (km 221,2) (Datenquelle: Amt der Tiroler Landesregierung, Sachgebiet Schutzwasserwirtschaft und Gewässerökologie)

verringern die Abfuhrkapazität und führen zur Überlastung von Schutzmaßnahmen bereits bei geringeren Abflüssen im Vorfluter. Analog gestaltet sich die Situation bei Retentionsräumen, wenn infolge von Anlandungen am Steuerpegel die Abflüsse überschätzt werden und eine frühzeitige oder $\mathrm{zu}$ intensive Dotierung des Retentionsraumes erfolgt. Hingegen können erhebli- che Sohleintiefungen die Dotierung von Retentionsräumen gänzlich verhindern, wenn die Abflüsse am Steuerpegel unterschätzt werden bzw. die lokalen Wasserspiegellagen die Überlaufkote des Einlaufbauwerkes nicht erreichen. Eine fundierte Einschätzung der Geschiebetransportprozesse im Hochwasserfall und der Stabilität der Gewässersohle ist in diesem Sinne für die Planung von
Hochwasserschutz- und Retentionsmaßnahmen von großer Bedeutung.

Der vorliegende Beitrag befasst sich mit der Bedeutung des Geschiebetransportes für die schutzwasserbaulichen Planungen am Inn im Unterinntal zwischen der Mündung der Sill bei Innsbruck und der Mündung der Weißache in Kufstein. Wesentliche Grundlagen sind neben den für die Geschiebemo- 


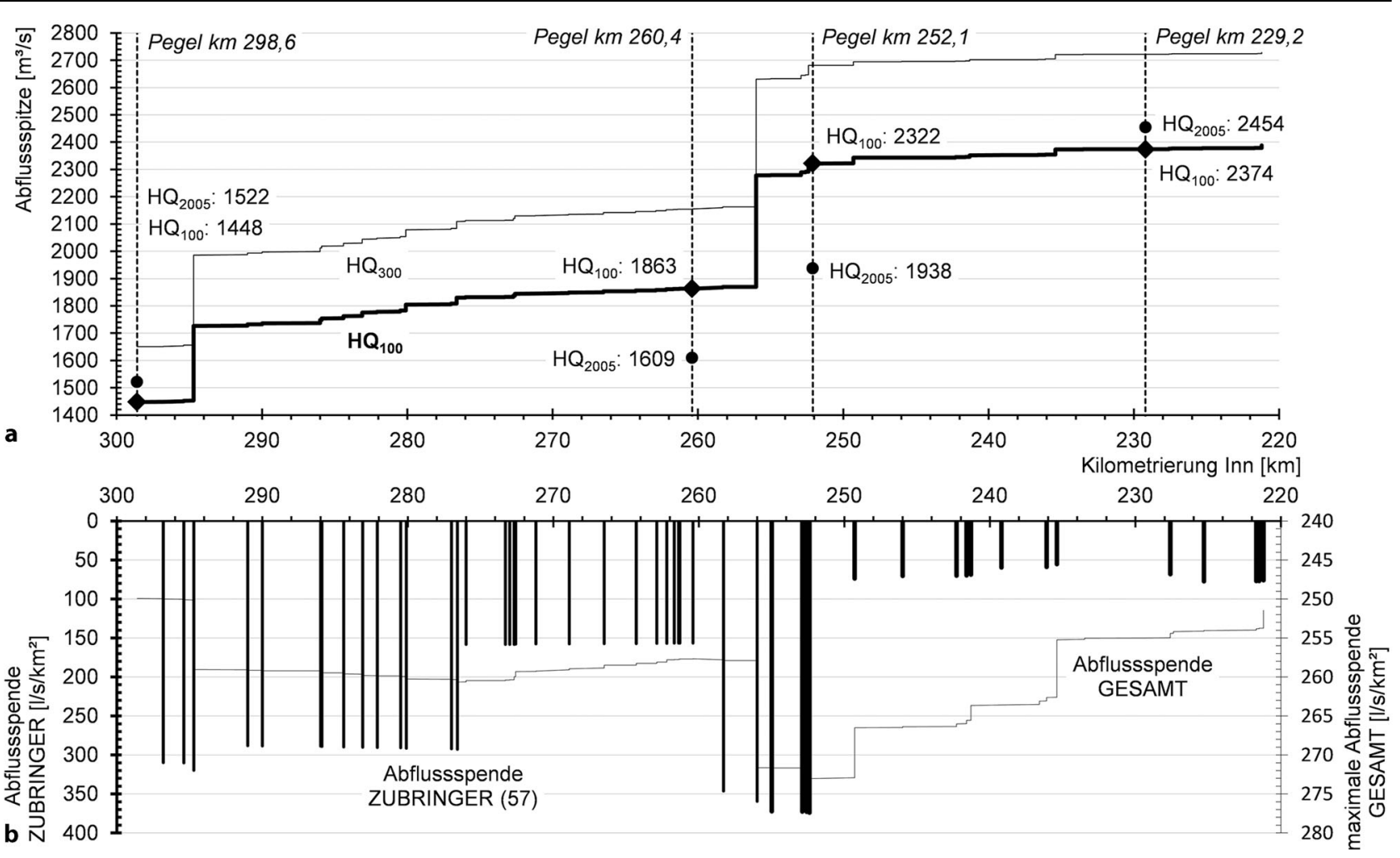

Abb. 3 (a) Hydrologischer Längenschnitt für den Innabschnitt von km 298,6 (Pegel Innsbruck/Inn) bis km 221,2 (Einmündung Weißache in Kufstein) mit den 100-jährlichen und den 300-jährlichen Bemessungswerten r; (b) Zubringerabflussspenden und Gesamtabflussspende $\left(\mathrm{HQ}_{100}\right)$ entlang des Inns

dellierung relevanten und in Kapitel 3 näher beschriebenen Erhebungen auch die Regionalstudie Unterinntal und der aktuelle Stand der Maßnahmenplanung für das Mittlere und Untere Unterinntal. Der 73,5 km lange Gewässerabschnitt ist nach der Harmonisierung der Hochwasserabflüsse im Jahr 2009 gegenwärtig nicht zur Gänze auf die schadlose Abfuhr eines 100-jährlichen Bemessungsereignisses ausgelegt. Die ausgewiesenen Gefahrenzonen (WRG 1959; BMLFUW 2014) für den Inn betreffen teilweise in erheblichem Maße bebaute und als Bauland gewidmete Flächen, landwirtschaftliche Flächen und bestehende Infrastruktur.

Umfangreiche schutzwasserwirtschaftliche Maßnahmen entlang des Inns, Maßnahmen zum Schutz von Siedlungen und Gewerbegebieten in überschwemmungsgefährdeten Gebieten und auch mehrere große Retentionsräume sind derzeit in Planung. Entlang der gesamten Gewässerstrecke und insbesondere am Ende des Gewässerabschnittes an der Staatsgrenze nach Deutschland gilt es, Abflussverschärfungen im Vergleich zur Bestandssituation zu vermeiden (vgl. Haider et al. 2014). Mit einer in diesem Kontext durchgeführten instationären 2Dnumerischen Geschiebemodellierung wird der Einfluss des Geschiebetransportes und sich einstellender Sohllagenänderungen auf die Planung der Hochwasserschutz- und Retentionsmaßnahmen aufgezeigt. Nachfolgend werden die numerischen Geschiebetransportberechnungen für längere $\mathrm{Ge}$ wässerabschnitte vorgestellt und die wesentlichen Erkenntnisse der Berechnungen für die Planung der technischen Schutzmaßnahmen am Inn aufgezeigt.

\section{Projektgebiet}

Abb. 2 zeigt den Gewässerabschnitt des Inns von der Einmündung der Sill bei Flusskilometer km 294,7 in Innsbruck bis zur Grenze zu Bayern bei Kufstein

Der Inn verläuft in diesem Gewässerabschnitt in einem über weite Strecken verbauten Gerinne mit durchwegs homogenem Querprofil. Das Gefälle beträgt im Durchschnitt etwa 1,2 \%0, die mittlere Sohlbreite $90 \mathrm{~m}$. 57 Zubringergewässer münden im Abschnitt von der Sillmündung in Innsbruck bis zur
Einmündung der Weißache in Kufstein in den Inn. Dabei vergrößert sich das Einzugsgebiet des Inns von $5.809 \mathrm{~km}^{2}$ um 63,5\% auf $9.497 \mathrm{~km}^{2}$. Der Inn passiert im Unterinntal 39 politische Gemeinden. Der gesamte Talraum ist geprägt durch eine intensive Flächennutzung für Wohnbau, Gewerbe und Landwirtschaft.

Die in Abb. 2 dargestellten Überflutungsflächen für ein 100-jährliches Hochwasserereignis zeigen, dass erhebliche Bereiche dieses Siedlungsraumes im unmittelbaren Überflutungsbereich des Inns liegen. Im Hochwasserfall stellen sie ein enormes Schadenspotenzial dar. Die dargestellten Überflutungsflächen bilden die Grundlage für die aktuelle Gefahrenzonenpläne für den Inn. Aufgrund der im Bestand streckenweise zu geringen Abfuhrkapazität des Inns und der daraus resultierenden Ausweisung der Gefahrenzonen ergeben sich bedeutende Einschränkungen und Konsequenzen für die Raumplanungsund Bebauungskonzepte der Gemeinden und das Entwicklungspotenzial von Gewerbe- und Industriebetrieben.

In Abb. 3 (a) ist der hydrologische Längenschnitt für den betrachteten $\mathrm{Ge}$ - 


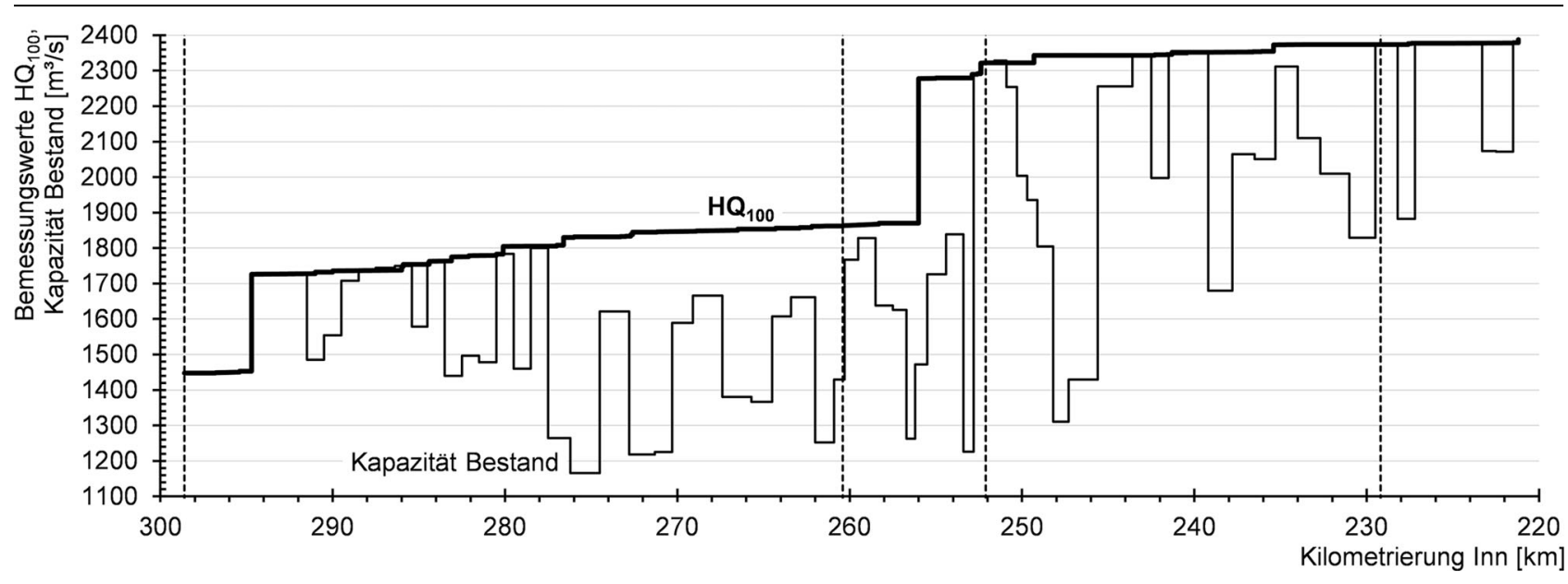

Abb. 4 Hydrologischer Längenschnitt für den Innabschnitt von km 298,6 bis km 221,2 mit den 100-jährlichen Bemessungswerten; Darstellung von Bereichen mit geringeren Abflusskapazitäten des Inns für die derzeitige Bestandssituation (Datenquelle hydrologischer Längenschnitt: Amt der Tiroler Landesregierung, Sachgebiet Schutzwasserwirtschaft und Gewässerökologie)

wässerabschnitt des Inns mit den 100jährlichen und den 300-jährlichen Bemessungswerten dargestellt. Die Werte repräsentieren in der Form die Einwirkung für die hydraulische Modellierung von oberstrom und aus den Zubringereinzugsgebieten für die hydraulische Modellierung. Die beiden markanten Zuflüsse erfolgen durch die Sill in Innsbruck $\left(274 \mathrm{~m}^{3} / \mathrm{s}\right.$ bei $\mathrm{HQ}_{100}$ des Inn) und den Ziller $\left(408 \mathrm{~m}^{3} / \mathrm{s}\right.$ bei $\mathrm{HQ}_{100}$ des Inn). Abb. 3 (b) enthält für den $\mathrm{HQ}_{100^{-}}$ Lastfall die durchwegs stationär angenommenen Abflussspenden aus den 57 Zubringereinzugsgebieten und die Gesamtabflussspende im Inn.

Im betrachteten Gewässerabschnitt des Inns sind vier Pegelmessstellen zur kontinuierlichen Wasserstandsaufzeichnung und Abflussermittlung situiert. Beim Hochwasser vom August 2005 wurde am Pegel in Innsbruck eine Abflussspitze mit einer Jährlichkeit $>200$ beobachtet. Flussabwärts nahm die Jährlichkeit ab (Abb. 3, (a)) (Godina et al. 2006). Das Hochwasser vom August 2005 verursachte in Tirol und dabei insbesondere im Tiroler Oberland (Rosanna, Trisanna, Sanna, Inn) verheerende Schäden (Godina et al. 2006). Im Unterland war insbesondere auch der westliche Bereich Wörgls auf der orografisch rechten Seite des Inns betroffen (vgl. Abb. 1 und Abb. 2). Das Hochwasser führte schließlich zu einer Erhöhung der 30-, 100- und 300-jährlichen Hochwasserbemessungswerte (Abb. 3 (a)).

Aufgrund der dargestellten Situation im Unterinntal erfolgen gegenwärtig im Auftrag der Bundeswasserbauver- waltung Tirol umfangreiche Planungsarbeiten für Hochwasserschutz- und Retentionsmaßnahmen im Mittleren Unterinntal von der ARGE WernerConsult/Henzinger/Freiland/Moser\&Jaritz und im Unteren Unterinntal von der ARGE DonauConsult/ILF. Diese umfassen Linearmaßnahmen zum Schutz des Siedlungsraums (insbesondere Dämme), mehrere große Retentionsräume im Talraum und auch technische Maßnahmen zum Schutz von Einzelobjekten. Abb. 4 zeigt den Verlauf der Abfuhrkapazität des Bestandsgerinnes (d. h. den maximalen Abfluss ohne Überflutungen, vgl. Abb. 2). Der Vergleich mit dem hydrologischen Längenschnitt gibt einen ersten Hinweis über das erforderliche Ausmaß an Linearmaßnahmen.

Derzeit ist die Anordnung von sechs teilweise gesteuerten Retentionsräumen in Thaur, Schwaz-Buch, Münster, Voldöpp (Kramsach), Radfeld-Kundl und Angath mit Volumina zwischen 1,2 Mio. $\mathrm{m}^{3}$ und 5,4 Mio. $\mathrm{m}^{3}$ zur Verhinderung nachteiliger Konsequenzen aus den Linearmaßnahmen für die Unterlieger im Inntal und auch für den weiteren Verlauf des Inns in Bayern vorgesehen. Die gesteuerte Dotierung der Retentionsräume basiert jeweils auf Beobachtungen eines Pegelkollektivs entlang des Inns und in den bedeutenden Zubringern, d. h. durch eine Echtzeit-Beobachtung der Wasserstände. Aufgrund der Charakteristik des Inns und der Erfahrungen aus abgelaufenen Hochwasserereignissen ist keine direkte Steuerung auf Basis eines Hochwasserprognosemodelles vorgesehen. Die Hochwasserschutz- und Retenti- onsmaßnahmen werden auf die 100jährlichen Bemessungswerte ausgelegt, zudem wird für den Überlastfall die Standsicherheit der Schutzbauwerke nachgewiesen (vgl. Haider 2014a).

Die Planungsarbeiten für die in Abb. 2 dargestellten Planungsräume Oberes, Mittleres und Unteres Unterinntal können aus hydraulischer Sicht unabhängig voneinander erfolgen. Nach Umsetzung der Maßnahmen ist schließlich eine Revision der ausgewiesenen Gefahrenzonen verbunden (WRG 1959; BMLFUW 2014).

Die folgenden Abschnitte befassen sich mit dem Einfluss des Geschiebetransports auf die Planung und Wirkung der Hochwasserschutz- und Retentionsmaßnahmen und zeigen exemplarisch Ergebnisse der 2D-numerischen Modellierung für einen Teilbereich des gesamten Planungsraums auf. Dargestellt wird der Abschnitt im Raum Brixlegg bis Kundl zwischen den Flusskilometern $\mathrm{km} \mathrm{251,1}$ und $\mathrm{km} \mathrm{240,0.}$ Die Maßnahmenplanung für diesen Abschnitt erfolgt von der ARGE DonauConsult/ILF und sieht unter anderem zwei über ein Pegelkollektiv gesteuerte Retentionsräume vor, jener in Voldöpp (Kramsach) orografisch links mit einem Volumen von 1,7 Mio. $\mathrm{m}^{3}$ und jener im Raum Radfeld-Kundl orografisch rechts mit einem Retentionsvolumen von 5,4 Mio. $\mathrm{m}^{3}$. Mit der Brandenberger Ache $\left(282,1 \mathrm{~km}^{2}\right)$ und der Wildschönauer Ache $\left(87,2 \mathrm{~km}^{2}\right)$ münden in diesem Abschnitt neben zahlreichen kleineren Zubringern auch zwei bedeutende und geschieberelevante Seitenzubringer in den Inn. 


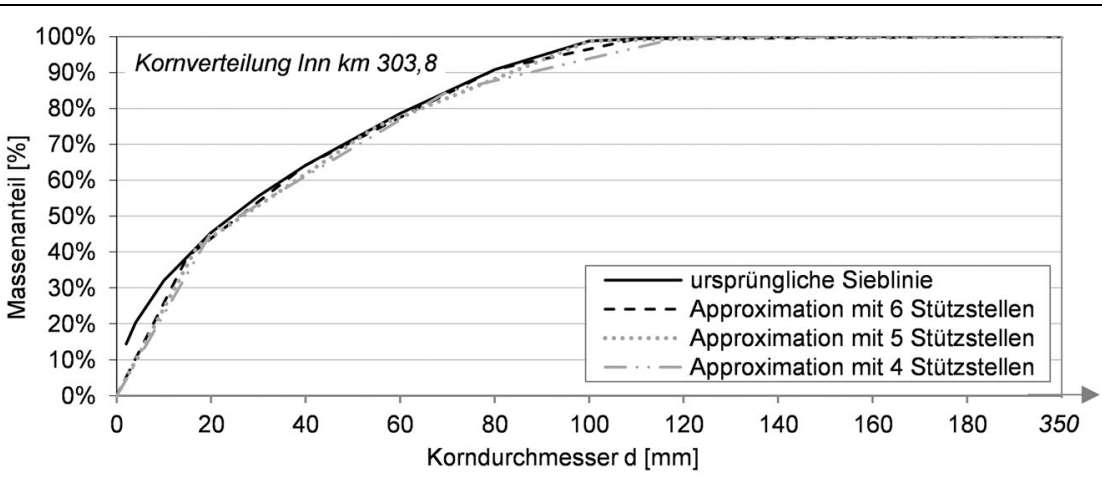

Abb. 5 Beispiel von Approximationen einer Kornverteilungskurve (KVK) mit verschiedenen Approximationsmethoden

\section{Methodik}

\section{1 Überblick}

Im vorliegenden Kapitel wird auf die wesentlichen Schritte und einflussreichsten (Modell-)Parameter bei der geschiebehydraulischen Modellierung näher eingegangen. Die Bearbeitung erfolgte in den folgenden fünf Arbeitsschritten: (i) Datenerhebung und -analyse, (ii) Bestimmung des Geschiebeeintrags durch den Inn am oberwasserseitigen Modellrand und die Seitenzubringer, (iii) Erstellung und Anpassung des numerischen Modells, (iv) Sensitivitätsanalyse für die maßgebenden Modellparameter und betrachtete Szenarien, (v) Simulationen und Ergebnisanalyse.

\subsection{Datenerhebung und -analyse}

Im Rahmen der Datenerhebung wurden in einer Kombination aus Linienzahlanalysen (LZA, $\mathrm{n}=21$ ) und Volumenproben $(n=20)$ notwendige Kenntnisse zur Sohlzusammensetzung des Inns und seiner Zubringer erhoben. Vor Ort wurden in Abhängigkeit von der Sohlenbeschaffenheit die Feststoffprobestellen ausgewählt. Bei einer vorhandenen Deckschicht wurde die Kornverteilung mittels Linienzahlanalyse an geeigneten Stellen im Flussbett (z. B. auf Kiesbänken) durchgeführt. In Bereichen ohne ausgeprägte Deckschicht bzw. nasser Probenahme erfolgte eine volumetrische Beprobung (Bunte und Abt 2001). In weiterer Folge wurden die Korngrößenverteilungen der Proben im Labor mittels Siebanalyse bestimmt. Anhand der aufgenommenen Einzelproben am Inn wurden hinsichtlich der Struktur der Gewässersohle homogene Teilabschnitte definiert. Die Korngrößenverteilungen der Deckschicht und
Unterschicht wurden für weitere statistische Auswertungen aufbereitet. Im Anschluss wurden die charakteristischen Korndurchmesser ausgewertet.

Bei einer numerischen Simulation mittels HYDRO_FT-2d müssen die Kornverteilungskurven approximiert werden. Die Wahl der Kornklassen und die Anzahl beeinflussen die numerische Berechnung. Umach (2013) beschäftigte sich in diesem Zusammenhang mit der Approximation von Kornverteilungskurven bei der zweidimensionalen numerischen Simulation von Geschiebetransportprozessen. Er stellte fest, dass eine Approximation mit maximal acht Kornklassen im Allgemeinen hinreichend genaue Berechnungsergebnisse liefert. Wenn Auflandungs- oder Umlagerungsprozesse dominieren, sind hingegen drei Stützstellen ausreichend. Mithilfe einer Sensitivitätsanalyse wurde versucht, eine günstige Approximation aller Kornverteilungskurven $\mathrm{zu}$ finden. Die größte Kornklasse ergab sich aus dem Maximalwert der Korngrößenverteilungen. Der Abgleich zwischen originaler und approximierter Verteilungslinie erfolgte anhand eines Vergleichs der charakteristischen Korndurchmesser und einem visuellen Vergleich (Abb. 5). Als charakteristische Korngrößen wurden der maßgebende Korndurchmesser $d_{\mathrm{m}}$, die 50\%-Korndurchmesserfraktile $d_{50}$ und die $90 \%$-Korndurchmesserfraktile $d_{90}$ ausgewertet. Tab. 1 gibt eine Übersicht der definierten Kornklassen, welche im numerischen Modell (Schichtenmanagement, Fraktionierung der Geschiebeeinträge) verwendet wurden. Hierbei steht $\mathrm{d}_{\mathrm{i} \text {,rep }}$ als das arithmetische Mittel der Kornklasse i und $d_{i}$ für den maximalen Korndurchmesser der Kornklasse i.

\subsection{Geschiebeeintrag durch den Inn} und die Seitenzubringer

Für die Berechnung der Geschiebetransportraten der Seitenzubringer bei einem Bemessungsereignis laut hydrologischem Längenschnitt mussten im ersten Schritt alle relevanten Zubringer bestimmt werden. Die Auswahl der relevanten Zubringer wurde anhand von zwei Kriterien vorgenommen: (i) der Zubringerabfluss im Verlauf des Bemessungsereignisses beträgt mindestens $10 \mathrm{~m}^{3} / \mathrm{s}$ und (ii) gemäß der verfügbaren Wildbachaufnahmeblätter ist ein nennenswerter Geschiebeeintrag in den Inn zu erwarten. Entsprechend diesen Erkenntnissen erfolgte die Definition aller geschieberelevanten Seitenzubringer. Für die Berechnung der Transportraten wurden Schlüsselstrecken definiert (Bezzola 2007). Dabei handelt es sich um transportlimitierende Strecken möglichst in unmittelbarer Nähe zu den Einmündungen in den Inn, welche ein geringes Gefälle aufweisen und den Weitertransport des Geschiebes begrenzen (siehe Abb. 6). Neben den vorhandenen Abflussganglinien wurden die gewässertypischen Parameter wie Gewässerbreite, Sohlgefälle, Böschungsneigung und der maßgebende Korndurchmesser $d_{m}$ bestimmt. Abb. 6 zeigt exemplarisch die beiden Schlüsselstrecken an der Sill und der Brandenberger Ache.

Die rechnerische Abschätzung des Geschiebeeintrages erfolgte mithilfe empirischer Berechnungen unter Berücksichtigung von Formverlusten. Auf Basis der Abflussganglinien, welche durch den hydrologischen Längenschnitt vorgegeben sind, konnte für jeden Zeitschritt die Geschiebetransportrate berechnet werden. Für die Abschätzung der Transportraten steiler Zubringer wurde der empirische Ansatz von Rickenmann (1990) und für große, flache Zubringer die Transportformel von Meyer-Peter und Müller (1949) verwendet. Für eine Plausibilisierung der Ergebnisse wurde ein Vergleich der Geschiebefrachten aus den empirischen Berechnungen mit den Informationen aus den Wildbachaufnahmeblättern angestellt.

Die spezifische Geschiebetransportkapazität $g_{\text {TK }}$ nach Rickenmann (1990) wird nach Gleichung (1) berechnet, jene nach Meyer-Peter und Müller (1949) ergibt sich aus Gleichung (2): 
Tab. 1 Kornklassen zur Charakterisierung der Gewässersohle des Inns und des eingetragenen Geschiebes

\begin{tabular}{|l|l|l|l|l|l|l|}
\hline Kornklassen/Fraktion i & $\mathbf{1}$ & $\mathbf{2}$ & $\mathbf{3}$ & $\mathbf{4}$ & $\mathbf{5}$ & $\mathbf{6}$ \\
\hline $\mathbf{d}_{\mathrm{i}, \text { rep }}[\mathbf{m m}]$ & 7,5 & 27,5 & 60 & 95 & 150 & 270 \\
\hline $\mathbf{d}_{\mathbf{i}}[\mathbf{m m}]$ & 15 & 40 & 80 & 110 & 190 & 350 \\
\hline$\Delta \mathbf{d}_{\mathbf{i}}[\mathbf{m m}]$ & 15 & 25 & 40 & 30 & 80 & 160 \\
\hline
\end{tabular}

$$
\begin{aligned}
g_{T K}= & 12,6 *\left(\frac{d_{90}}{d_{30}}\right)^{0,2}\left(q_{\text {spe }}-q_{\text {spec }, c}\right) * \\
& I_{\text {red }}^{2} *(s-1)^{-1,6} \\
g_{T K}= & \sqrt{(s-1) * g} * d_{m}^{3 / 2} * 8 * \\
& \left(\theta^{\prime}-\theta_{c}\right)^{3 / 2}
\end{aligned}
$$

gen für Masse und Impuls. Die Diskretisierung erfolgt auf Basis eines FiniteVolumen-Schemas mit explizitem Zeitschrittverfahren. Der Geschiebetransport wird mit der fraktionierten Formel von Meyer-Peter und Müller (1949), adaptiert von Hunziker (1995), welche im Besonderen das Transportverhalten von weitgestuften Kiesflüssen beschreibt, berechnet (siehe Gleichung 2). Die Berechnung basiert auf einem Schichtenkonzept zur Charakterisierung der Gewässersohle, welche die Definition einer Austauschschicht (AS), einer Unterschicht (US) und einer Grundschicht (GS) erfordert. Die Dicke der Austauschschicht $\left(d^{\mathrm{ex}}\right)$ stellt einen für das gesamte Berechnungsgebiet und die gesamte Berechnungsdauer konstanten Modellparameter dar. Dieser wird in der Regel mit Werten zwischen $d_{90}$ und dem Vierfachen des $d_{90}$ der KVK angegeben und definiert jenen Bereich der Gewässersohle, der unmittelbar vom transportierten Geschiebe beeinflusst wird bzw. mit diesem in Austausch steht. Die darunter liegende Unterschicht wird durch eine variable (von der jeweils vorherrschenden KVK abhängige) Schichtdicke gekennzeichnet. Die Grundschicht wird entsprechend den geologischen Gegebenheiten angegeben. Die mit dem Geschiebetransport einhergehende Sohllagenänderung im numerischen Modell wird über die Exner-Gleichung (Exner 1925) erfasst.

Im Rahmen der Untersuchungen steht die Betrachtung der Auswirkungen der Sohllagenänderungen auf die geplanten Hochwasserschutzmaßnahmen, im Speziellen auf die Retentionsräume, im Mittelpunkt. Modelliert wird das 100-jährliche Bemessungsereignis (laut hydrologischem Längenschnitt) für drei verschiedene Szenarien jeweils für die Zustände vor (IST-Zustand) und nach Errichtung der Linear- und Retentionsmaßnahmen (PLAN-Zustand). Bei den drei Szenarien handelt es sich um verschiedene Parametereinstellungen, welche eine Bandbreite der zu erwartenden Ergebnisse darstellen und damit den generellen Unsicherheiten in der Geschiebetransportmodellierung Rechnung tragen (siehe Kapitel 3.4).
Als Basis für die Simulationen dienten die vom Sachgebiet Schutzwasserwirtschaft und Gewässerökologie zur Verfügung gestellten hydraulischen Modelle aus der Regionalstudie Unterinntal vom Büro Pieler $\mathrm{ZT} \mathrm{GmbH}$ und der Maßnahmenplanung von der ARGE WernerConsult/Henzinger/Freiland/ Moser\&Jaritz und der ARGE DonauConsult/ILF. Aufgrund der erheblichen Größe des Untersuchungsgebietes und des im Allgemeinen sehr hohen Rechenaufwands von fraktionierten Geschiebetransportberechnungen wurde das Modell in sechs Teilmodelle unterteilt. An den Modellrändern wurden Überlappungsbereiche bzw. Vorlaufstrecken angeordnet. Hinsichtlich der numerischen Stabilität bei den Simulationen war es notwendig, die Berechnungsnetze zu optimieren. Dies erfolgte durch eine geringfügige Glättung einiger Übergangsbereiche Sohle-Böschung bzw. Böschung-Vorland und der Adaptierung der Netzelemente zu Vierecks-Elementen. Die geometrischen Veränderungen führten zu geringfügigen Wasserspiegellagenänderungen in der Reinwasserbetrachtung, welche im Weiteren durch Anpassung der Manning-Strickler-Beiwerte $\left(\mathrm{k}_{\mathrm{St}}\right)$ behoben wurden.

In der weiteren Umgebung von Abflussverschärfungen, hervorgerufen durch Linearmaßnahmen, werden zur Hochwasserscheitelsenkung Retentionsräume geplant. Die Genauigkeit der Prognose inklusive der Lage der Retentionsräume $\mathrm{zu}$ den Steuerpegeln ist von entscheidender Bedeutung (Fischer 2008). Die ungesteuerten Retentionsräume werden durch Streichwehre, welche im Modell in Form geometrisch überströmbarer Elemente nachgebildet wurden, beaufschlagt. Bei gesteuerten Retentionsräumen werden die Verschlüsse an den Einlaufbauwerken über eine Wasserstand-AbflussBeziehung geregelt. Im numerischen Modell konnte der Einlauf in den Retentionsraum über eine „Auslauf W-Q/ Wehr"-Beziehung an einen vordefinierten Steuerpegel gekoppelt werden, welcher sich naturgemäß in einem Bereich mit beweglicher Sohle befindet.

Für die numerische Berechnung müssen Randbedingungen an sämtlichen Modellrändern gesetzt werden (ÖWAV 2007). An den Zulaufrändern wurden die Abflussganglinien $\left[\mathrm{m}^{3} / \mathrm{s}\right]$ aus dem hydrologischen Längenschnitt, die Geschiebeeinträge als Sedigraphen $[\mathrm{kg} / \mathrm{s}]$ mit den berechneten Fraktions- 


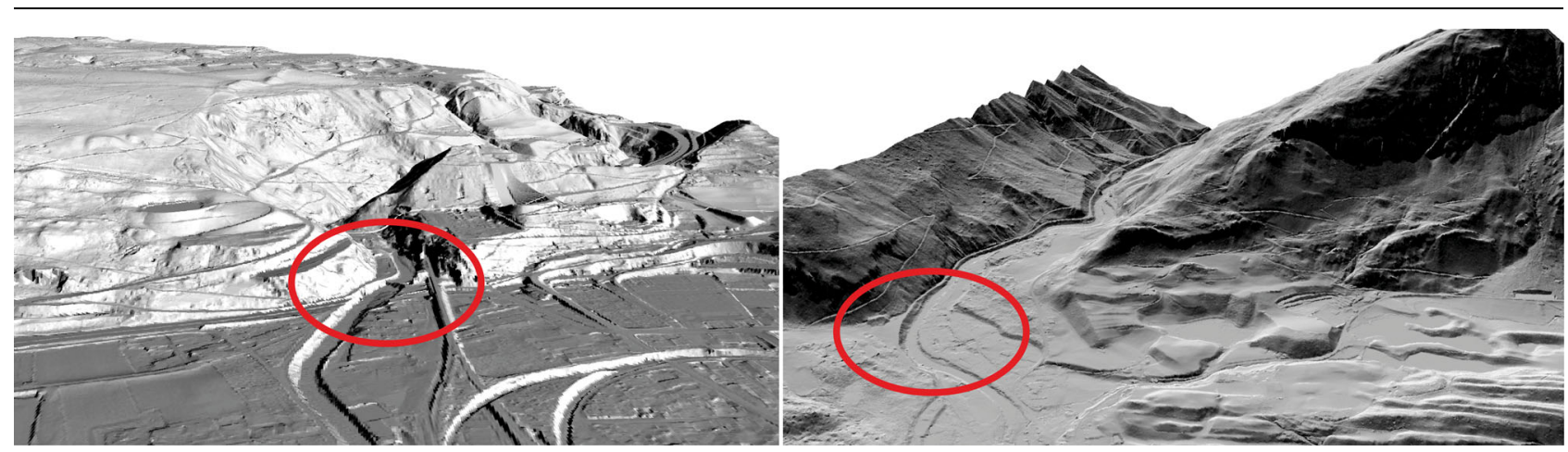

Abb. 6 Darstellung ausgewählter Schlüsselstrecken (links: Sill, rechts: Brandenberger Ache; vgl. Abb. 2)

anteilen $\mathrm{p}_{\mathrm{i}}[\%]$ angesetzt. Im Hinblick auf die numerische Stabilität der Simulationen und die Vermeidung unrealistischer Anlandungen unmittelbar an den Zulaufrändern wurden die Ganglinien im numerischen Modell auf mehrere hintereinander angeordnete "Nodestrings“ aufgeteilt. Am Auslaufrand definiert das Energieliniengefälle die Randbedingung. Als hydraulische Anfangsbedingung wurde zusätzlich eine Wasserspiegelhöhe bei geringen Abflüssen im gesamten Flussschlauch definiert. Damit können unrealistische Geschiebeumlagerungen am Berechnungsbeginn vermieden werden. Die Rauheiten nach Strickler (Gesamtwert) wurden aus dem hydraulischen Modell übernommen. Die Formrauheit $\mathrm{k}_{\mathrm{st}, \mathrm{r}}$ wurde mit der Formel von Wong und Parker (2006) ermittelt.

Die aufgenommenen Korngrößenverteilungen des Flussbettes bilden die Grundlage für den Sohlaufbau im Sedimenttransportmodell. Als Ergebnis einer Sensitivitätsanalyse wurden zwei unterschiedliche Dicken (Szenarien 1 und 2) der Austauschschicht mit $\mathrm{d}^{\mathrm{ex} 1}=0,20 \mathrm{~m}$ und $\mathrm{d}^{\mathrm{ex} 2}=0,10 \mathrm{~m}$ festgelegt (vgl. Kapitel 4). Die Fraktionsanteile der einzelnen Schichten jeweils zu Berechnungsbeginn resultieren aus den Kornverteilungen entlang des Inns und wurden für jeden Netzknoten definiert. Die maximale Erosionstiefe für die Gewässersohle wurde mit einem Meter begrenzt $\left(D Z_{\max }=1 \mathrm{~m}\right)$. Im Bereich von Brücken, Modellrandbedingungen wie Einlauf-, Stoffeintrags- und Modellauslaufbereichen wurde die Erosion unterbunden.
4 Sensitivitätsanalyse und Szenarien - Plausibilisierung von gewählten Modellparametern und Berechnungsergebnissen

Aufgrund nicht ausreichend verfügbarer Messdaten und Beobachtungen zum Geschiebetransport am Inn, insbesondere bei Hochwassersituationen, besteht keine Möglichkeit einer gezielten geschiebehydraulischen Kalibrierung. Mit diesem Hintergrund wird zunächst eine Sensitivitätsanalyse der maßgebenden Modellparameter durchgeführt. Im Rahmen dieses Untersuchungsschrittes können kritische modell- und gewässerspezifische Parameter variiert und deren Einfluss auf die Berechnungsergebnisse analysiert werden. Ausgehend von einer Ausgangssituation wurde der Einfluss von neun Modellparametern an einem Teilmodell untersucht. Ziel dieser Sensitivitätsanalyse war es letztlich, für die Modellierung des gesamten Gewässerabschnittes plausible und belastbare Modellparameter und Modellansätze ansetzen zu können und den Einfluss des Geschiebetransportes auf die Wirkung der Hochwasserschutz- und Retentionsmaßnahmen und die damit verbundenen Unsicherheiten beurteilen zu können. Es wurde unter anderem der Einfluss der in Tab. 2 angeführten modell- und gewässerspezifischen Parameter untersucht.

Über die Dicke der Austauschschicht $\left(d^{\mathrm{ex}}\right)$ kann die Geschwindigkeit von Erosions- und Anlandungsprozessen gesteuert werden. Bei der Wahl einer geringen Austauschschichtdicke kommt es insbesondere bei der Berechnung von Hochwasserabflüssen zu raschen Veränderungen, gegebenenfalls zum „Aufreißen“ der Deckschicht und dadurch bedingt zur Mobilisierung der Feststoffe aus der Unterschicht.
Bereiche mit stabiler (statischer) Deckschicht können im numerischen Modell durch Definition größerer Austauschschichtdicken abgebildet werden (Klar 2016).

Der Bewegungsbeginn wird anhand des kritischen Shields-Parameters $\left(\theta_{\mathrm{c}}\right)$ erfasst und entsprechend den Untersuchungen von Meyer-Peter und Müller (1949) mit $\theta_{c}=0,047$ festgelegt. Jüngeren Untersuchungen beispielsweise von Wong und Parker (2006) und Tritthart et al. (2011) zufolge lässt sich der kritische Shields-Parameter als (innerhalb eines realistischen Bereiches) variabler Parameter beschreiben, der durchaus vom ursprünglich abgeleiteten Wert abweichen kann und grundsätzlich im Rahmen numerischer Modellierungen als Kalibrierparameter $\mathrm{zu}$ betrachten ist. Die Sensitivitätsanalyse für das Teilmodell des Inns belegt, dass durch geringere $\theta_{\mathrm{c}}$-Werte der Transportbeginn früher einsetzt. Ein signifikanter Einfluss auf die Sohllagenentwicklung während des 100-jährlichen Bemessungshochwassers wurde allerdings nicht beobachtet.

Meyer-Peter und Müller (1949) beschreiben in ihrem Formelansatz den Formelskalierfaktor $c_{\mathrm{MPM}}$ und definieren diesen mit dem Wert 8 (Wiberg und Smith 1989; Hunziker 1995; Wong und Parker 2006). Durch die Variation des Formelskalierfaktors konnte gezeigt werden, dass höhere Werte für $c_{\text {MPM }}$ die berechneten Transportkapazitäten naturgemäß erhöhen und folglich die sich einstellenden Erosionsbereiche und -tiefen vergrößern.

Die Berücksichtigung des lokalen Ungleichgewichts vom theoretischen Transportvermögen $q_{\mathrm{B}}{ }^{*}$ zum tatsächlichen Transport $q_{\mathrm{B}}$, welches z. B. bei einem Übergang von einer abgepflasterten $\mathrm{zu}$ einer erodierbaren Sohle herrscht, kann mit der Einführung der Anpassungslänge $L_{S}$ berücksichtigt 


\begin{tabular}{|c|c|c|}
\hline Modellspezifische Parameter & Parameter & Einfluss \\
\hline Schichtenmanagement & $\begin{array}{l}p a l=0,37(\hat{=} 10 \mathrm{~cm})-1,00(\hat{=} 27 \mathrm{~cm}) \\
p u l=4,00(\hat{=} 54 \mathrm{~cm})-14,00(\hat{=} 70 \mathrm{~cm})\end{array}$ & $\nearrow$ \\
\hline $\begin{array}{l}\text { kritischer } \\
\text { Shields-Parameter }\end{array}$ & $\begin{array}{l}\theta_{c}=0,047 \\
\theta_{c}=0,035\end{array}$ & $\rightarrow$ \\
\hline Formelskalierfaktor & $k_{\mathrm{F}}=0,625\left(\hat{=} C_{\mathrm{MPM}}=5\right)-k_{\mathrm{F}}=1,5\left(\hat{=} C_{\mathrm{MPM}}=12\right)$ & $\nearrow$ \\
\hline Anpassungslänge & $\begin{array}{l}\alpha_{\mathrm{L}}=0-9000 \\
L_{S 0}=0-50\end{array}$ & $\rightarrow$ \\
\hline Gewässerspezifische Parameter & Parameter & Einfluss \\
\hline Geschiebeeintrag Seitenzubringer & $\begin{array}{l}\text { Einkorn Formelansatz Meyer-Peter und Müller (1949) * } \\
\text { Fraktionierter Formelansatz in HYDRO_FT-2d * } \\
\text { * verschiedene KGV }\left(\mathrm{d}_{\mathrm{m}}=16 \mathrm{~mm}, \mathrm{~d}_{\mathrm{m}}=18 \mathrm{~mm}\right)\end{array}$ & $\searrow$ \\
\hline $\begin{array}{l}\text { Situierung Geschiebeeintrag Seitenzu- } \\
\text { bringer } \\
\text { (Lage der Nodestrings) }\end{array}$ & $\begin{array}{l}\text { in Flussmitte } \\
\text { im Seitenzubringer (Mündungsbereich) } \\
\text { im Flusslauf - seitliche Positionierung }\end{array}$ & $\nearrow$ \\
\hline
\end{tabular}

werden. Phillips und Sutherland (1989) haben mit experimentellen Untersuchungen eine Formel dieses „Loading Law Konzeptes“ entwickelt, welche in HYDRO_FT-2d implementiert ist. Durch die Anpassung des $\alpha_{\mathrm{L}}$-Koeffizienten (Default: $\alpha_{\mathrm{L}}=4000$ ) und der minimalen Anpassungslänge $L_{\mathrm{so}}=15 \mathrm{~m}$ (Default) konnte das Ungleichgewicht in den Übergangsbereichen abgefangen und die Anlandungen über größere Bereiche „verschmiert" werden.

Neben dem Einfluss modellspezifischer Parameter auf die Berechnungsergebnisse wurden auch gewässerspezifische Parametern untersucht, $u$. a. der Einfluss des eingetragenen Geschiebes an den Zulaufrändern des numerischen Modells. Zwei Formelansätze (siehe Tab. 2) unter Zugrundelegung unterschiedlicher Korngrößenverteilungen wurden betrachtet. Es zeigte sich, dass das am Zulaufrand des Inns zugegebene Geschiebe während des Hochwasserereignisses den Auslauf des Teilmodelles nicht erreicht. Daraus kann geschlossen werden, dass der Modellaustrag im Wesentlichen das Sohlmaterial umfasst bzw. vom Schichtenmanagement und den gewählten KVKs bestimmt wird. Die Wahl des Formelsatzes zur Berechnung der Geschiebeeinträge hat hierfür eine geringe Bedeutung. Diese Feststellung unterstreicht die Bedeutung des Schichtenmanagements.

Die grobkörnigen Geschiebeeinstöße aus den Zubringern zeichnen sich durch Ablagerungskörper im Mündungsknoten im Vorfluter aus. Damit diese realitätsgetreu im Modell abgebildet werden können, wurde die Anordnung der „Nodestrings“ für die Geschiebeeinträge untersucht. Durch die seitliche Positionierung normal zur Fließrichtung konnte die kegelartige
Ablagerung am besten abgebildet werden. Die Lage der Zuflüsse hat lokal keinen maßgebenden Einfluss auf die Wasserspiegellagen.

Als Ergebnis der Sensitivitätsanalyse wurden drei Szenarien definiert, welche ein Spektrum der im Hochwasserfall $\mathrm{zu}$ erwartenden Sohllagenänderungen abbilden und mit welchen die morphodynamischen Änderungen für IST- und PLANzustand analysiert werden. Folgender Parametersatz wurde für alle Simulationen übernommen: $\theta_{\mathrm{c}}=0,047 ; k_{\mathrm{st}}=$ jeweils zeitlich konstant mit Werten entsprechend der Reinwasserkalibrierung; $\alpha_{\mathrm{L}}=4000$ und $L_{\mathrm{so}}=15$. Für Szenario 1 ergab sich eine Parameterkombination von pal $=0,74$ $\left(d^{\mathrm{exl}}=20 \mathrm{~cm}\right)$ und $k_{\mathrm{F}}=1,0$. Für Szenario 2 wurde sowohl die Dicke der Austauschschicht $\left(d^{\mathrm{ex} 2}=10 \mathrm{~cm}\right)$ als auch der Formelskalierfaktor $\left(k_{\mathrm{F}}=1,25\right)$ verändert. Für Szenario 3 wurden die Parametereinstellungen aus Szenario 1 übernommen, jedoch die Geschiebeeinträge aus den Seitenzubringern mit dem Faktor 1,4 multipliziert, sodass die Auswirkungen größere Seiteneinträge analysiert werden konnten.

\section{Ergebnisse}

\subsection{Korngrößenanalyse und Geschiebetransportraten}

Anhand der Korngrößenverteilungen aus allen Beprobungen entlang des Inns und einer visuellen Beurteilung der Sohlstruktur wurden vier homogene Teilabschnitte entlang des Inns definiert. Die Auswertung in Abb. 7 zeigt, dass die Korngrößenverteilungen mit zunehmender Fließlänge abnehmen. Im Bereich der Innschleife bei Kirchbichl km 232,3 wurden die ge- ringsten Korngrößen erhoben. Es ist allerdings darauf hinzuweisen, dass durch die Wasserkraftwerke Kirchbichl (Wehrbauwerk bei km 233,4) und Langkampfen (Wehrbauwerk bei km 223,5) das Sedimentkontinuum unterbrochen ist und somit ein Einfluss auf die Korngrößen und Schichten im Gewässerbett zu erwarten ist. Im dargestellten Längenschnitt ist in manchen Bereichen ein Gegengefälle erkennbar, welches auf lokale Kolk- und Anlandungsbereiche bzw. die Auswertung anhand der offiziellen Gewässerachse, welche nicht zwangsläufig dem Talweg entspricht, zurückzuführen ist. Die maßgebenden Korndurchmesser der Deckschicht liegen zwischen $2,0 \mathrm{~cm}$ und $4,0 \mathrm{~cm}$ und der Unterschicht zwischen $1,3 \mathrm{~cm}$ und $3,2 \mathrm{~cm}$. Die durchgeführten Analysen der Korngrößenverteilungen offenbaren, dass das Sohlmaterial am Inn (Modellbeginn) relativ grob ist und für das laufende Geschiebe nicht geeignet ist. Daher wurde für die Berechnung des Geschiebeeintrages die Korngrößenverteilung von Langkampfen $\mathrm{km} \mathrm{223,1}$ $\left(d_{\mathrm{m}, \mathrm{US}}=16 \mathrm{~mm}\right)$ zugrunde gelegt. Die charakteristischen Korndurchmesser der Seitenzubringer weisen eine hohe Variabilität auf. Der maßgebende Korndurchmesser streut zwischen $1,4 \mathrm{~cm}$ bis $7,9 \mathrm{~cm}$. Es ist keine Tendenz hinsichtlich Geologie bzw. Abflussverhältnissen (Einzugsgebietsgröße) zu erkennen.

Auf Basis der Korngrößenanalysen der Seitenzubringer wurde die Ereignisgeschiebefracht abgeschätzt und mithilfe der Wildbachaufnahmeblätter auf ihre Plausibilität geprüft. Die Datenblätter unterscheiden sich vom angewandten Lastfall dahingehend, dass die geschätzten Wildbachfrachten von einem $\mathrm{HQ}_{150}$-Ereignis ausgehen. Die Ergebnisse zeigten dennoch recht gute 

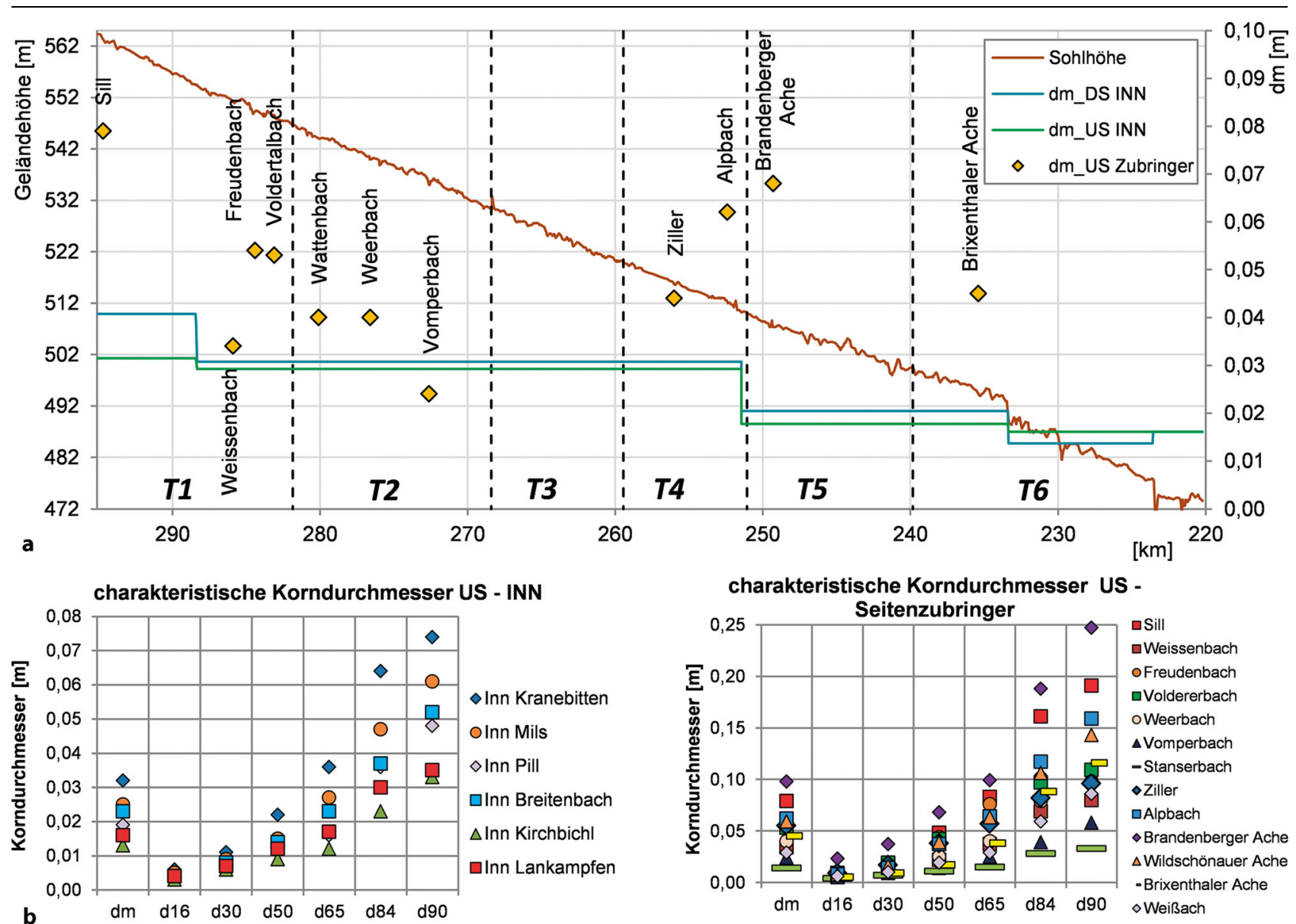

Abb. 7 (a) Maßgebende Korndurchmesser $d_{m, U s}$ und $d_{m, D s}$ entlang der untersuchten Innstrecke und der geschieberelevanten Zubringer; (b) maßgebende Korndurchmesser $d_{m}$, us der Unterschicht entlang des Inns; (c) maßgebende Korndurchmesser $d_{\mathrm{m} \text {,us }}$ der Seitenzubringer (Datenquelle Längenschnitt: hydraulisches Modell - Amt der Tiroler Landesregierung, Sachgebiet Schutzwasserwirtschaft und Gewässerökologie)

Übereinstimmungen bei den angenommenen Abflussganglinien; am Wattenbach und Haberbach wurden auf die Wildbachfrachten verwendet. In Tab. 3 sind die geschieberelevanten Zubringer mit den dazugehörigen Abflüssen laut hydrologischem Längenschnitt sowie den berechneten Ereignisgeschiebefrachten dargestellt.

Die Geschiebefracht am Zulaufrand von Teilmodell T1 des Inns wurde mit der Formel von Meyer-Peter und Müller (1949) berechnet. Für die Gesamtsimulation erfolgte eine durchgehende Berechnung mit einer direkten Übertragung der Ergebnisse vom oberstrom liegenden Auslaufquerschnitt zum $\mathrm{Zu}$ laufrand des nächsten Teilgebietes. Grundlagen zur Plausibilisierung der Eintragsfrachten waren Geschiebestudien von Hofer (1990), welche Auskunft über die Jahresgeschiebefrachten der Perioden 1952 bis 1987 geben. Die Ereignisfracht am Zulaufrand von Teilmo- dell T5 des Inns beträgt $23.831 \mathrm{~m}^{3}$ im ISTzustand und entspricht etwa $1 / 10$ der mittleren Jahresgeschiebefracht vom $60 \mathrm{~km}$ flussab gelegenen Untersuchungsstandort in Kirchbichl. In Abb. 8 sind die Zuflussganglinie (schwarze Linie), der Sedigraph (braune Linie) und die dazugehörigen Fraktionsanteile in \% der jeweiligen Kornklassen des untersuchten Teilmodells T5 dargestellt.

\subsection{Wirkung von Hochwasserschutz- und Retentionsmaßnahmen in der Reinwasserbetrachtung}

Lineare Hochwasserschutzmaßnahmen verursachen eine Abflussverschärfung. Diese negativen Auswirkungen auf den Hochwasserscheitel sind zu kompensieren (Haider 2014b). Durch die Anordnung von derzeit sechs Retentionsmaßnahmen kann die Abflussverschärfung welche aus den geplanten Linearmaß- nahmen resultiert, beim betrachteten Bemessungsereignis mit ,idealer' Steuerung ausgeglichen werden. Unter ,ideale' Steuerung wird die Ausnutzung des Bemessungsrückhaltevolumens und eine horizontale Kappung der Hochwasserwelle verstanden. In der Regionalstudie Unterinntal (PLAN-Zustand) wurde ein ungesteuerter Retentionsraum (Thaur) geplant, welcher mit einer fixen Überlaufkante berücksichtigt ist. In der Reinwasserbetrachtung kann eine optimale Scheitelreduktion erreicht werden; dies ist jedoch abhängig vom Hochwasserwellenverlauf. Des Weiteren werden derzeit in der Maßnahmenplanung für das Mittlere und Untere Unterinntal fünf über Pegelkollektive gesteuerte Hochwasserrückhaltemaßnahmen mit Dotationsbauwerken vorgesehen, welche die Abflussverschärfung aufgrund der Linearmaßnamen ausgleichen sollen. In diesem Beitrag näher behandelt sind 
Tab. 3 Geschieberelevante Zubringer mit zugehöriger Abflussspitze laut hydrologischem Längenschnitt und berechneter Ereignisgeschiebefracht

\begin{tabular}{|l|l|l|l|}
\hline Zubringer & Flkm & $\begin{array}{l}\text { Abflussspitze } \\
{\left[\mathrm{m}^{3} / \mathrm{s}\right]}\end{array}$ & $\begin{array}{l}\text { Geschiebefracht } \\
{\left[\mathrm{m}^{3}\right]}\end{array}$ \\
\hline Sill & 294,7 & 278,0 & 5400 \\
\hline Weissenbach & 285,9 & 12,0 & 2850 \\
\hline Freudenbach & 284,4 & 15,0 & 3560 \\
\hline Voldertalbach & 283,1 & 12,0 & 3131 \\
\hline Wattenbach & 280,1 & 22,0 & 5000 \\
\hline Weerbach & 276,6 & 25,0 & 3797 \\
\hline Vomperbach & 272,6 & 15,0 & 1605 \\
\hline Ziller & 256,0 & 408,0 & 336 \\
\hline Haberbach & 252,9 & 11,8 & 5000 \\
\hline Alpbach & 252,4 & 30,5 & 7688 \\
\hline Brandenberger Ache & 249,3 & 20,0 & 123 \\
\hline Brixenthaler Ache & 235,4 & 18,4 & 740
\end{tabular}

die beiden Retentionsräume Voldöpp (Dotation bei km 249,0) und RadfeldKundl (Dotation bei km 247,7), welche sich im dargestellten Untersuchungsgebiet (Teilmodell T5) befinden. Diese Retentionsräume werden über Wasserstandsinformationen an einem Pegelkollektiv gesteuert. Aus modelltechnischen Gründen wurde im Gerinne nur ein Steuerpegel angebracht, welcher mit dem Einlaufbauwerk gekoppelt ist. In der Reinwasserbetrachtung wurden die Wasserstand-Abfluss-Beziehungen so definiert, dass die Retentionsräume im Falle des 100-jährlichen Bemessungsereignisses optimal ausgenutzt werden. Der Retentionsraum Voldöpp erreicht somit ein Retentionsvolumen von max. 1,7 Mio. $\mathrm{m}^{3}$ und führt $\mathrm{zu}$ einer Wellenscheitelsenkung im Inn um $71 \mathrm{~m}^{3} / \mathrm{s}$ (siehe Abb. 9). Die Linearmaßnahmen entlang des Retentionsraumes führen dennoch $\mathrm{zu}$ einer Abflussverschärfung, welche durch den unterstrom gelegenen Retentionsraum kompensiert wird. Der Retentionsraum
Radfeld-Kundl fasst ein Volumen von max. 5,4 Mio. $\mathrm{m}^{3}$ und führt bei ,idealer Steuerung $\mathrm{zu}$ einer Scheitelreduktion von $209 \mathrm{~m}^{3} / \mathrm{s}$, was wieder die bedeutende Wirkung der Retentionsmaßnahmen hervorhebt.

In Abb. 10 sind die maximalen Überflutungsflächen des IST- und PLANZustands aus der hydrodynamischen Modellierung für Teilmodell T5 zu sehen. Durch die gezielte Beaufschlagung der Retentionsräume werden Vorländer vor Überflutungen geschützt. Die Ausdehnungen der Überflutungsflächen reduzieren sich maßgeblich. Landwirtschaftlich genutzte Flächen in den Retentionsräumen werden teilweise $\mathrm{zu}$ einem späteren Zeitpunkt geflutet als im Zustand vor Umsetzung der Maßnahmen. Durch die geplanten Retentionsräume kann eine erfolgreiche Rückhaltewirkung erzielt und dem Verschlechterungsverbot in vollem Umfang Rechnung getragen werden.

Der Vergleich der Ergebnisse aus der Reinwassermodellierung mit jenen der Geschiebetransportberechnungen ermöglicht eine Einschätzung der Auswirkungen des Geschiebetransports auf die Wirksamkeit der Hochwasserschutz- und Retentionsbauwerke.

\subsection{Auswirkung der Geschiebedynamik auf die geplanten Hochwasserschutzmaßnahmen}

\subsubsection{Volumenbilanzierung und Geschiebeaustrag}

Bei der technischen Planung von Hochwasserschutz- und Retentionsmaßnahmen wird der Einfluss des Geschiebetransports in Betracht gezogen. Die Flussmorphologie (Erosions- und Sedimentationsprozesse) steht in einer Wechselwirkung mit dem Abflussverhalten eines Gewässers bei Hochwasser und kann zu Problemen bei der Hochwasserschutzplanung führen (Habersack et al. 2013). In Bezug auf die zeitliche und räumliche Entwicklung der Sohllagenänderung entlang des Inns wurden auf Basis der geschiebehydraulischen Modellierungen Volumenvergleiche angestellt. Abb. 11 zeigt das Ergebnis der Umlagerungen in Teilmodell T5 für jeden Kilometer nach Durchlauf der Hochwasserwelle. Dargestellt sind alle drei Szenarien des Bestandszustands und der Maßnahmenplanung. Am Modellrand Teilmodell T5 wird ein Gesamtvolumen von ca. $21.100 \mathrm{~m}^{3}$ eingetragen und das Austragsvolumen beträgt ca. $32.200 \mathrm{~m}^{3}$. Es kommt zu einer Volumenänderung im Teilmodell $\mathrm{T} 5$ von rund $11.600 \mathrm{~m}^{3}$. Die Ergebnisse der Volumenbilanzierung lassen jedoch keine ausgeprägte (Szenario-unabhängige) Erosions- bzw. Anlandungstendenz über den gesamten Abschnitt erkennen. Die Abwei-
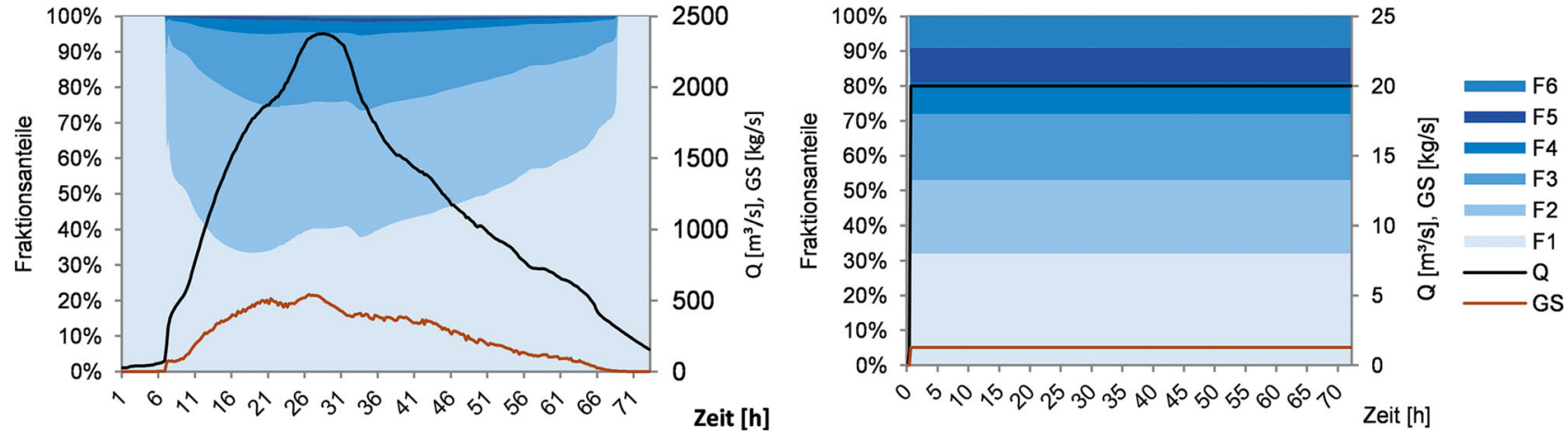

Abb. 8 Teilmodell T5-Zuflussganglinie $Q\left[\mathrm{~m}^{3} / \mathrm{s}\right]$ mit berechnetem Sedigraphen GS $[\mathrm{kg} / \mathrm{s}]$ und deren Fraktionsanteile $\mathrm{p}_{\mathrm{F} 1-\mathrm{F} 6}[\%]$ für den Zulaufrand von Teilmodell T5 am Inn (links) und für die Brandenberger Ache (rechts) 


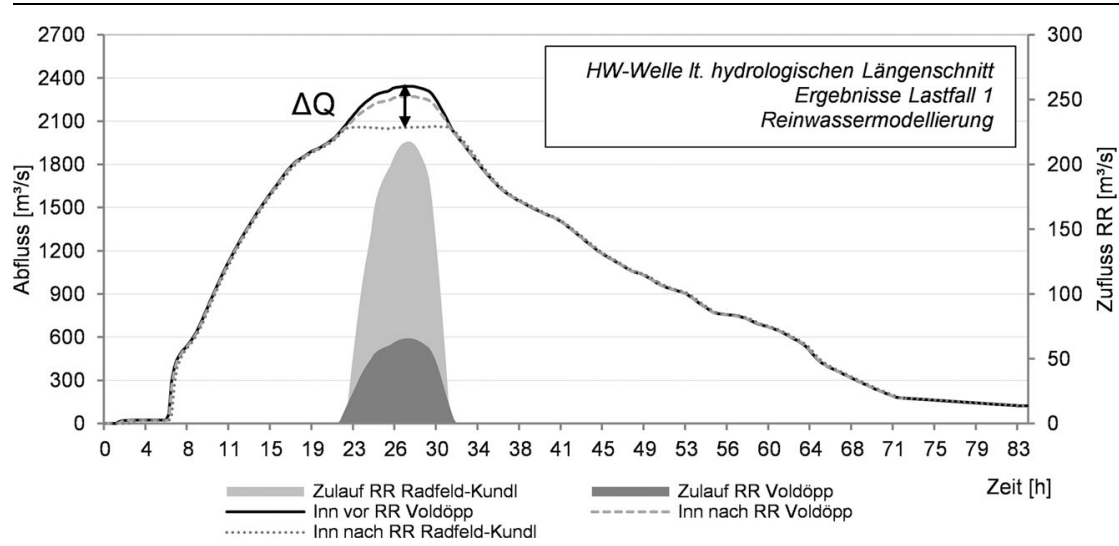

Abb. 9 Teilmodell T5-Scheitelreduktion $\Delta Q$ durch die beiden Retentionsräume Voldöpp und Radfeld-Kundl sowie Zuflüsse in die Retentionsräume während des 100jährlichen Bemessungsereignisses

chungen zwischen IST- und PLANZustand können durch die vorgenommenen Maßnahmen begründet und als sensible Bereiche betrachtet werden. In Abschnitt 3 (km 249,5 bis km 248,5) sind relativ hohe Anlandungstendenzen bis $\mathrm{zu} 8.720 \mathrm{~m}^{3} \mathrm{zu}$ erwarten. Dieser Bereich weist bedingt durch die Einmündung der Brandenberger Ache und die damit verbundene verhältnismäßig große Gewässerbreite geringe Transportraten auf. Durch den Retentionsraum Voldöpp wird die morphologische Umlagerung im Vergleich zum IST-Zustand reduziert. Im darauffolgenden Kilometer kommt es im Mittel
5.4 Wirkung von Hochwasserschutzund Retentionsmaßnahmen unter

Berücksichtigung des

Geschiebetransports

Die Wirkung von Retentionsmaßnahmen ist neben den hydrologischen Zuständen von verschiedenen Eigenschaften der Retentionsräume und von der Steuerung abhängig (Haider 2014b). Eine wichtige Voraussetzung für eine optimale Ausnutzung der Retentionsräume ist eine relativ stabile Sohle im Bereich der Steuerpegel. Mit der Sohllagenänderung ist eine Wasserspiegellagenänderung im Gewässer und somit eine Änderung der Wasserstand-Abfluss-Beziehung verbunden, welche für die Steuerung benötigt wird. In der Praxis ist es schwer möglich, eine stabile Sohle im Bereich der Steuerpegel natürlich oder baulich herzustellen, daher ist die Berücksichtigung des Geschiebetransports in der Planung von maßgebender Bedeutung.

Im Folgenden sind die Ergebnisse hinsichtlich Sohllagenänderung für IST- und PLAN-Zustand im Längenschnitt (Aufnahme an einem Punkt in der Gewässerachse) illustriert (siehe Abb. 12). Neben der Ausgangssohllage (braune Linie) sind die Sohllagendifferenzen der drei Szenarien mit und ohne Maßnahmen gegenübergestellt. Die umhüllenden in grau dargestellten

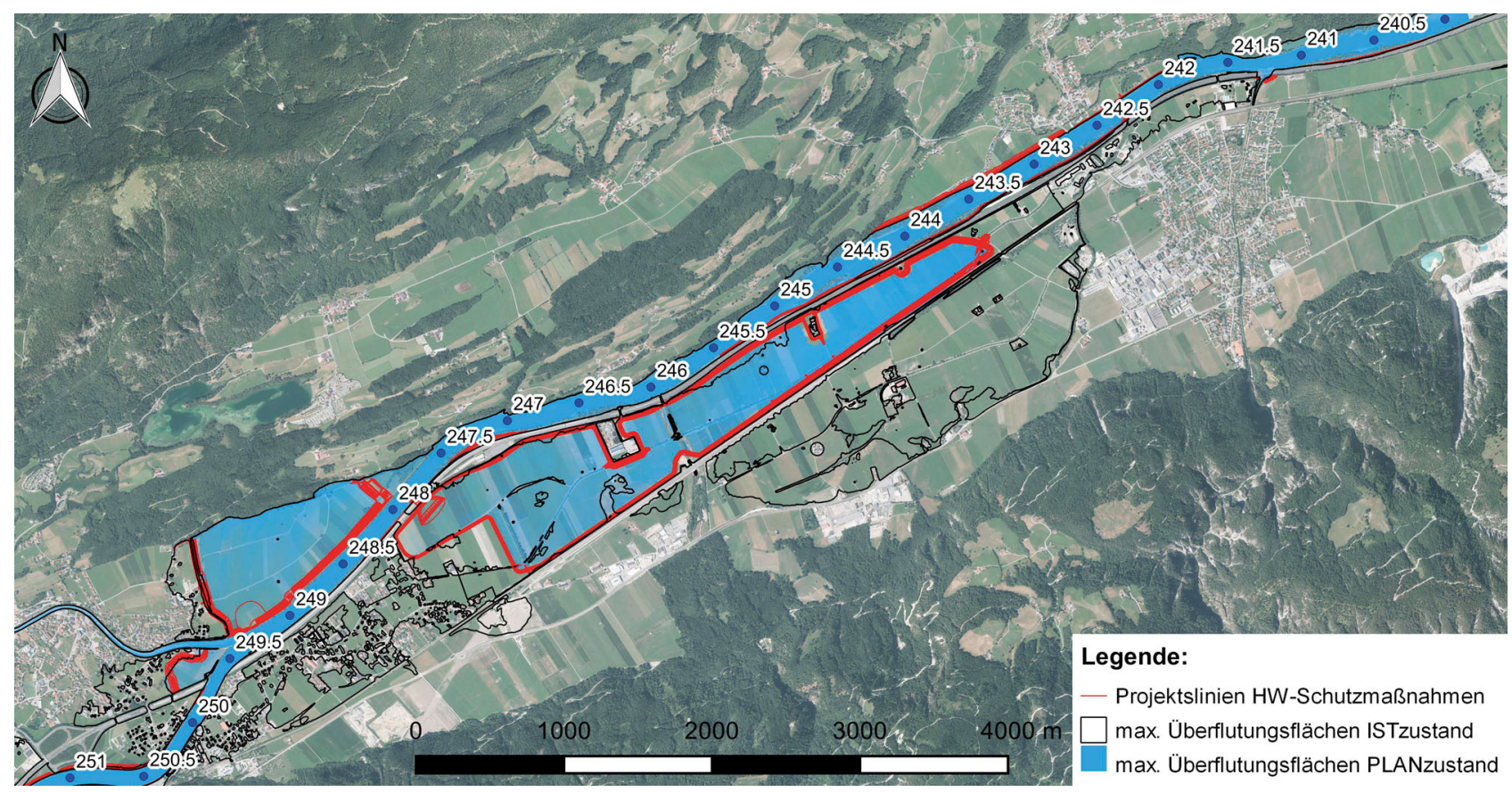

Abb. 10 Teilmodell T5-Vergleich der maximalen Überflutungsflächen von IST- (schwarze Umrandungslinie) zu PLAN-Zustand (blaue Fläche) aus der hydrodynamischen Modellierung 


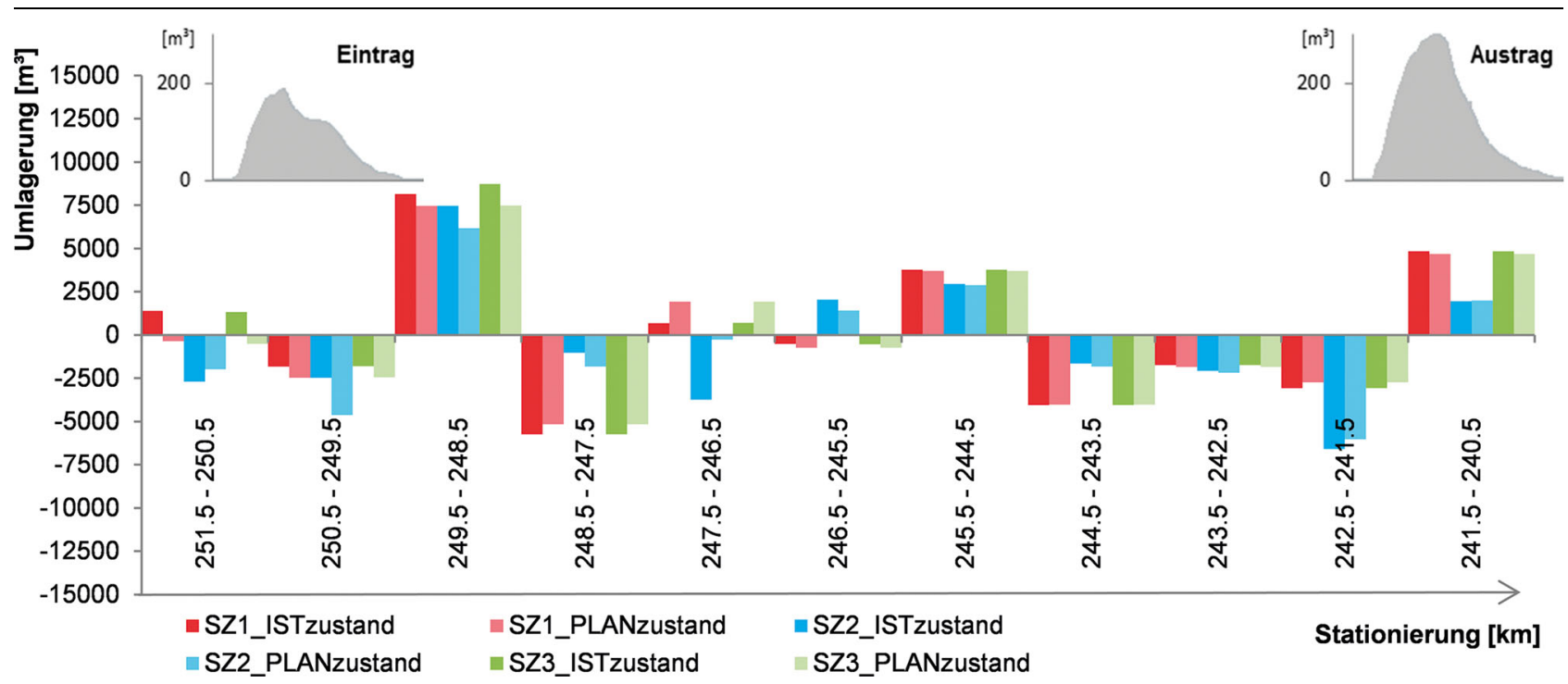

Abb. 11 Teilmodell T5-Umlagerungen aller Szenarien (vgl. Kapitel 3.3) für IST- und PLAN-Zustand (Einlaufbereich RR Voldöpp km249, 1 bis km 248,9, Einlaufbereich RR Radfeld-Kundl km 247,8 bis km 247,6)

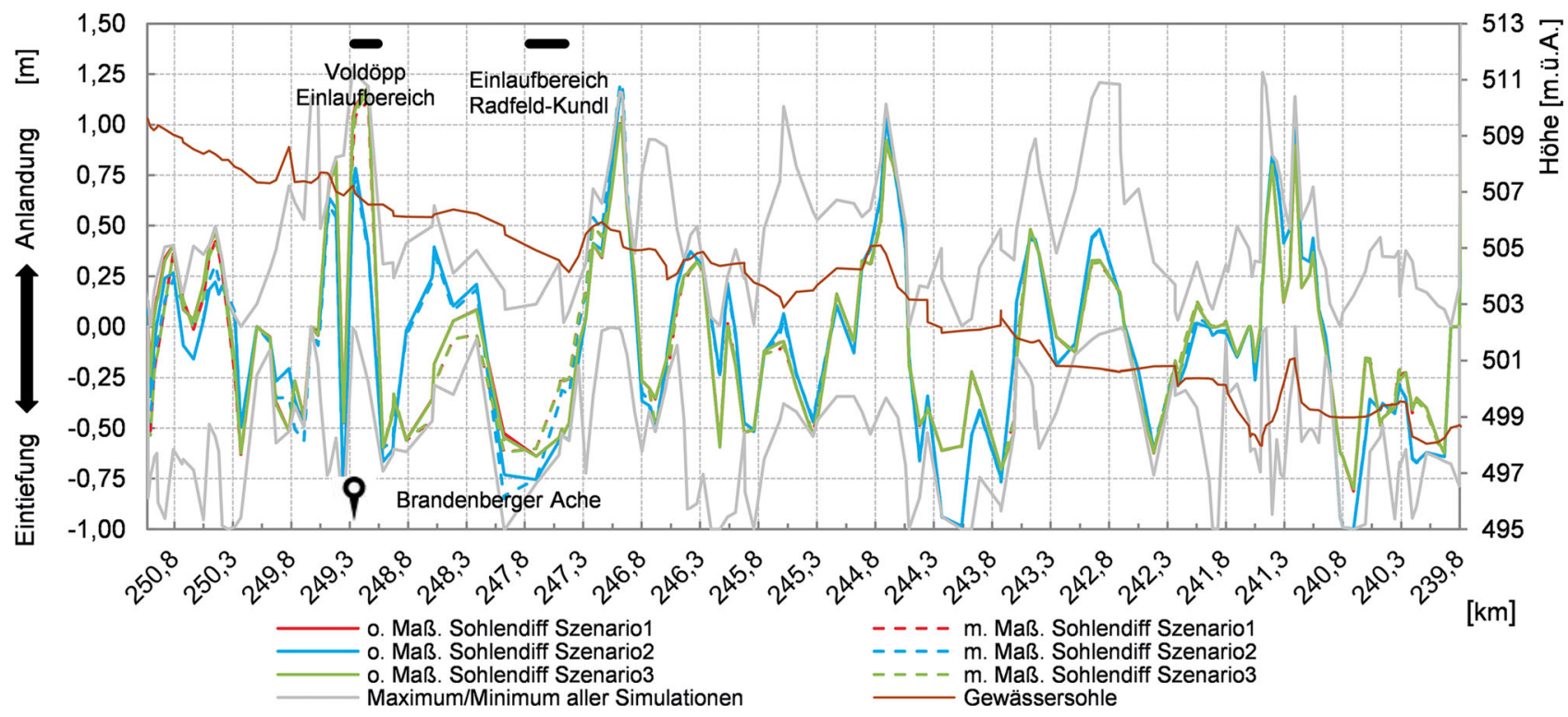

Abb. 12 Teilmodell T5-Differenz der Sohllagen zu den Zeitpunkten vor und nach dem 100-jährlichen Bemessungshochwasser; Auswertung im Längenschnitt (Aufnahme an einem Punkt in der Gewässerachse); strichlierte Linien symbolisieren die Sohllagenänderungen imPLAN-Zustand, diegrauen Linien stellen die minimalen und maximalen Differenzen imzeitlichen Verlauf der Simulationen aller drei Szenarien für den IST-Zustand dar

Linien deuten auf die minimalen und maximalen Sohlhöhenänderungen aller Simulationen über den gesamten zeitlichen Verlauf des Hochwassers.

In Abb. 13 sind die Sohllagenänderungen (Szenario 1) am Ende der Simulation und die maximalen Überflutungsflächen dargestellt. Die durch die simulierte Hochwasserwelle auftretende Sohllagenänderung schwankt zwischen $+1,25 \mathrm{~m}$ und $-1,00 \mathrm{~m}$, wobei die höchsten Anlandungen in strömungsberuhigten Bereichen, wie z. B. in Buhnenfeldern, bzw. in breiten Gewässerabschnitten mit geringem Gefälle vorkommen. Der Szenarienvergleich führt vor Augen, dass es bei unterschiedlichen Parametereinstellungen zu unterschiedlichen Simulationsergebnissen kommt, die grundsätzliche Ausdehnung von Erosions- bzw. Anlandungsbereichen bleibt hingegen unverändert. Im Vergleich zu den Szenarien 1 und 3 wurde bei Szenario 2 (blaue Linie) eine geringe Austauschschichtdicke $\left(d^{\text {ex }}=10 \mathrm{~cm}\right)$ angesetzt. Dies führt zu erhöhten Erosionstiefen in belasteten Bereichen und einer feineren Kornverteilung der Austauschschicht. Bei Szenario 3 werden die Geschiebeeinträge um den Faktor 1,4 höher angesetzt. Durch die erhöhten Geschiebezugaben aus den Seitenzubringern konnte lokal grundsätzlich keine nennenswerte Änderung in der Sohllage festgestellt werden. Einzig bei der Betrachtung der Wasserspiegellagen werden lokale Änderungen hervorgerufen, welche jedoch auf den geplanten Hochwasserschutz 


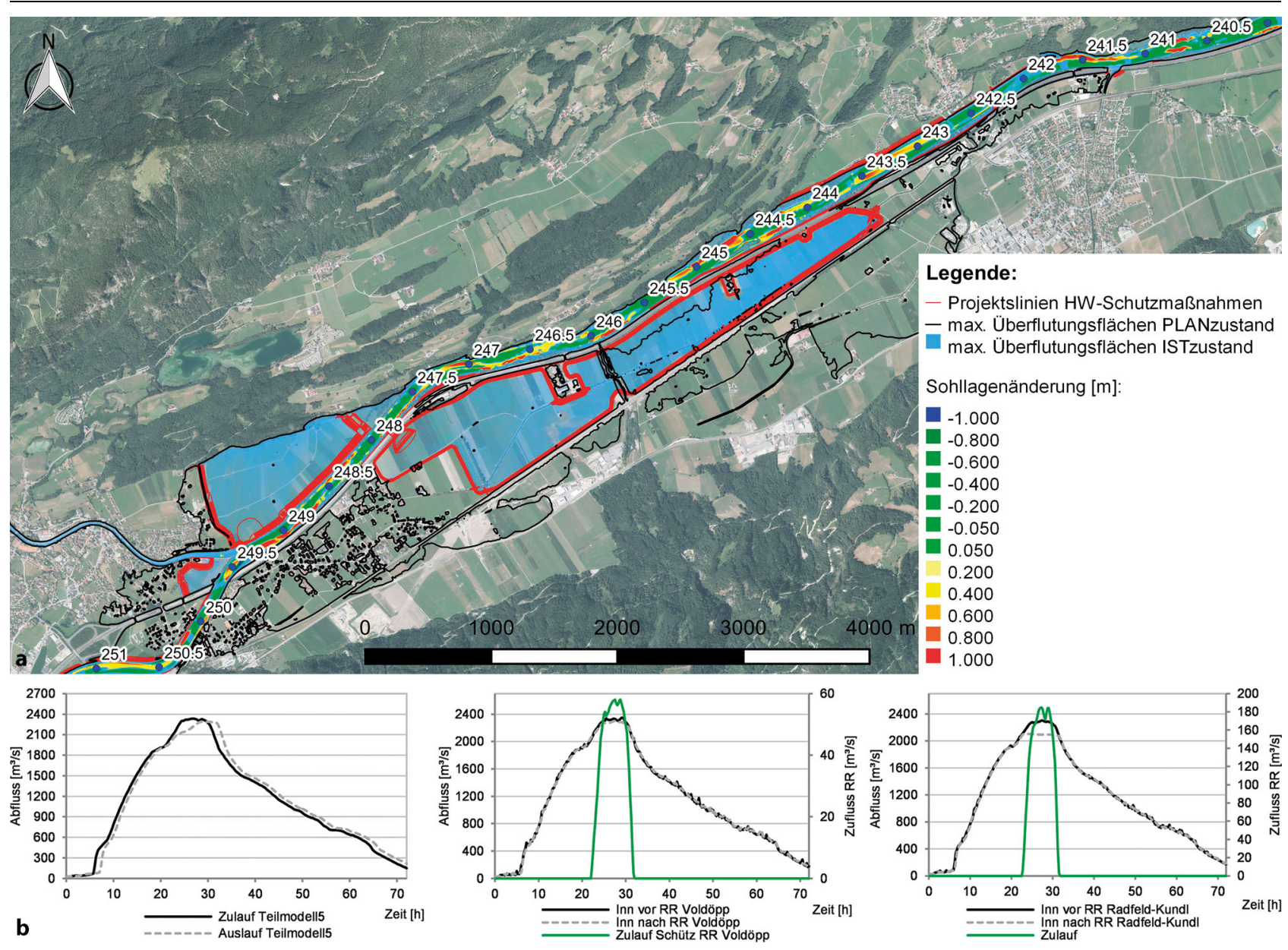

Abb. 13 Teilmodell T5-Darstellung des Endzustands der Sohllagenänderung (Szenario 1); Vergleich der maximalen Überflutungsflächen von IST-Zustand (schwarze Umrandungslinie) und PLAN-Zustand (blaue Fläche) aus der hydrodynamischen Modellierung

keine signifikanten Auswirkungen haben.

Der Steuerpegel für beide Retentionsräume wurde am Modellrand ca. bei km 250,8 eingerichtet. Der Einfluss des Modellrandes muss mitberücksichtigt werden. Die Simulation zeigt, dass der Geschiebetransport negative Effekte auf die Dotierung der Retentionsräume hat. Im Bereich des Pegelpunktes kommt es primär zur Erosion und im abfallenden Hochwasserwellenast gleicht sich die Sohle wieder aus. Durch die Sohllagenänderung verändert sich der Wasserstand und liegt ca. $6 \mathrm{~cm}$ tiefer als bei der Reinwassermodellierung. Die Konsequenz daraus ist, dass die Retentionsräume $\mathrm{zu}$ einem späteren Zeitpunkt dotiert werden. Die Dotation des Retentionsraumes Voldöpp (Einlauf bei km 249,0) erfolgt zwischen $22,25 \mathrm{~h}$ und $32,00 \mathrm{~h}$ und erreicht statt eines dimensionierten Retentionsvolumens von 1,7 Mio. $\mathrm{m}^{3}$ nur 1,5 Mio. $\mathrm{m}^{3}$. Aus dem Ergebnis geht hervor, dass der Retentionsraum nicht optimal ausgenutzt wird. Unter Berücksichtigung des Geschiebetransports wird mit der Dotierung des Retentionsraumes eine Wellenscheitelreduktion von $2.347 \mathrm{~m}^{3} / \mathrm{s}$ auf $2.301 \mathrm{~m}^{3} / \mathrm{s}$ erreicht, das entspricht $1,96 \%$ der in diesem Bereich auftretenden Hochwasserspitze (nach dem Retentionsraum). Der maximale Wasserstand im Retentionsraum Voldöpp beträgt ca. 3,5 m und liegt im nördlichen Randbereich des Rückhalteraumes. In der Detailbetrachtung des Einlaufbereichs ist ersichtlich, dass bis zum Zeitpunkt der Dotation ( $\mathrm{t}=22,25 \mathrm{~h}$ ) die Sohllagenänderungen in IST- und PLAN-Zustand ähnlich sind. Bei Öffnung des Einlaufbauwerkes wird die Abflussfracht reduziert und ruft unmittelbar nach dem Entnahmebauwerk eine Anlandung hervor. Dieser Prozess ist bei allen Parametereinstellungen zu erkennen.

Durch die räumlich geringeren Überflutungsflächen wirken lineare Hoch- wasserschutzmaßnahmen abflussverschärfend und führen $\mathrm{zu}$ einer Beschleunigung der Welle. Der unterstrom gelegene Retentionsraum Radfeld-Kundl wurde auf eine Größe von 5,2 Mio. $\mathrm{m}^{3}$ dimensioniert und erstreckt sich über $4,5 \mathrm{~km}$. Es sei noch einmal darauf hingewiesen, dass die Veränderung der Sohllage beim Steuerpegel von Bedeutung ist. Unter Berücksichtigung des Geschiebetransports wird eine Abflussfracht von 4,4 Mio. $\mathrm{m}^{3}$ in den Retentionsraum geleitet, in der Reinwasserbetrachtung werden 5,2 Mio. $\mathrm{m}^{3}$ zurückgehalten. Dieser Unterschied ist darin begründet, dass durch die Sohlerosion auch der Wasserstand entsprechend absinkt und die WasserstandAbfluss-Beziehung nicht mehr optimal für den Retentionsraum ausgelegt ist. Der maximale Zufluss in den Retentionsraum reduziert sich von $218 \mathrm{~m}^{3} / \mathrm{s}$ auf $185 \mathrm{~m}^{3} / \mathrm{s}$. In Bezug auf die Bandbreitenbetrachtung der einzelnen Lastfälle zeigte das Modellergebnis, dass 
bei Lastfall 2 das maximale Füllvolumen von 4,1 Mio. $\mathrm{m}^{3}$ erreicht wird. Die Begründung hierfür liegt wiederum in den Parametereinstellungen, welche stärkere Erosionsprozesse zulassen. Eine ähnliche Sohlentwicklung wie beim Einlaufbereich Voldöpp ist auch beim Retentionsraum RadfeldKundl zu erwarten. Durch das große Fassungsvolumen des Retentionsraums reduzieren sich die Abflussfracht, die Wassertiefe des Inns und somit die einwirkende dimensionslose Schubspannung. Nichtsdestotrotz wird durch den optimierten Retentionsraum Radfeld-Kundl der Wellenscheitel am Inn in erheblichem Maße reduziert.

Aus der Simulation geht hervor, dass der Auslauf im angeführten Projektabschnitt (Teilmodell T5) um $45 \mathrm{~m}^{3} / \mathrm{s}$ reduziert wird und Ausuferungen verhindert werden. Die Anschlagslinien sind in diesem Teilbereich deckungsgleich mit den Ergebnissen aus der Reinwasserbetrachtung. Die Maßnahmen schützen alle nahegelegenen Siedlungs- und Gewerbegebiete und tragen somit zur Verbesserung der Hochwassersituation bei.

\section{Diskussion}

Zur Vermeidung bzw. Verminderung zukünftiger Hochwasserschäden am Inn im Unterinntal ist die Umsetzung von Linear- und Retentionsmaßnahmen unabdingbar. Die Anwendung einer geschiebehydraulischen Modellie- rung und die Kenntnis der dafür erforderlichen Daten und Parameter (Sohlstruktur, Geschiebeeinträge, Modellparameter etc.) erlaubt die Untersuchung der Sohllagenentwicklung und damit des Einflusses des Geschiebetransportes auf die Maßnahmenplanung. Eine möglichst verlässliche Vorhersage der Sohllagenänderung erfordert eine umfassende Datengrundlage sowie auch die Kalibrierung und Validierung des numerischen Modells. In diesem Kontext konnte - aufgrund der fehlenden Datengrundlagen zur Modellkalibrierung - mit dem aus einer Sensitivitätsanalyse hervorgehenden drei betrachteten Szenarien ein Spektrum der im Hochwasserfall zu erwartenden Sohllagenänderungen dargestellt werden.

Es zeigt sich, dass die Dynamik der Gewässersohle für die Steuerung der Retentionsräume von maßgebender Bedeutung ist, sodass aus ihr wesentliche Informationen über die maximale Sohllagenänderung abgeleitet werden können. Um eine erfolgreiche Ausnützung der Überflutungsräume $\mathrm{zu}$ ermöglichen, ist sowohl im Nahbereich der Steuerpegel als auch bei den Einlaufbauwerken in die Retentionsräume eine möglichst stabile Sohle erforderlich bzw. muss die mögliche Schwankung bei der Dimensionierung der Einlaufbauwerke berücksichtigt werden. Durch die räumlich und zeitlich veränderliche Sohllage wird beispielsweise im Erosionsfall der kritische Wasserstand am Steuerpegel zu einem späte- ren Zeitpunkt erreicht. Folglich werden die Retentionsräume später dotiert und letztlich nicht optimal ausgenutzt. Aus den Untersuchungen geht die Wichtigkeit dieser Problematik hinsichtlich einer optimalen Ausnutzung der Retentionsräume hervor. Dieses Projekt trägt zum Konzept der Risikominimierung der Planungsarbeiten der Hochwasserschutzmaßnahmen am Inn im Unterinntal bei. Die Modellierungsergebnisse in Form maximal zu erwartender Sohllagenänderungen und deren Auswirkungen auf den Hochwasserschutz werden in die Detailplanung einfließen und stellen eine besondere Herausforderung für Auftraggeber/innen und Planer/innen dar.

Open access funding provided by University of Innsbruck and Medical University of Innsbruck.

Open Access Dieser Artikel wird unter der Creative Commons Namensnennung 4.0 International Lizenz (http:// creativecommons.org/licenses/by/4.

0/deed.de) veröffentlicht, welche die Nutzung, Vervielfältigung, Bearbeitung, Verbreitung und Wiedergabe in jeglichem Medium und Format erlaubt, sofern Sie den/die ursprünglichen $\mathrm{Au}$ tor(en) und die Quelle ordnungsgemäß nennen, einen Link zur Creative Commons Lizenz beifügen und angeben, $o b$ Änderungen vorgenommen wurden.

\section{Literatur}

ARGE DonauConsult/ILF (2016): Hochwasserschutz Tiroler Unterinntal - Maßnahmenplanung Unteres Unterinntal, Vorentwurf, Auftraggeber Bundeswasserbauverwaltung Tirol (nicht veröffentlicht).

ARGE WernerConsult/Henzinger/Freiland/ Moser\&Jaritz (2016): Hochwasserschutz Tiroler Unterinntal - Maßnahmenplanung Mittleres Unterinntal, Vorentwurf, Auftraggeber Bundeswasserbauverwaltung Tirol (nicht veröffentlicht).

Aufleger, M., Gems, B., Klar, R. (2012): Flussaufweitungen als flussbauliche Methode - Grundsätze und Werkzeuge. Österreichische Wasserund Abfallwirtschaft 64 (7-8): 363-378.

Bätzing, W. (1991): Die Alpen: Entstehung und Gefährdung einer europäischen Kulturlandschaft. In: Beck C H. München.

Bezzola, G. R. (2007): Ereignisanalyse Hochwasser 2005. 1. Prozesse, Schäden und erste Einordnung. Bundesamt für Umwelt BAFU, Bern.

Bundesministerium für Land- und Forstwirtschaft, Umwelt und Wasserwirtschaft (2014): WRG-Gefahrenzonenplanungsverordnung

WRG-GZPV, BGBl. II Nr. 145/2014. Wien.

Bundesministerium für Land- und Forstwirtschaft, Umwelt und Wasserwirtschaft (2015):
Nationaler Hochwasserrisikomanagementplan RMP 2015. GZ:BMLFUW-IL.99.1.1/0191IV/2015. Wien.

Bunte, K., Abt, S. R. (2001): Sampling surface and subsurface particle-size distributions in wadable gravel- and cobble-bed streams for analyses in sediment transport, hydraulics, and streambed monitoring. U.S. Dept. of Agriculture, Forest Service, Rocky Mountain Research Station.

Exner, F. M. (1925): Über die Wechselwirkung zwischen Wasser und Geschiebe in Flüssen. Sitzungsberichte der Akademie der Wissenschaften in Wien. Abt. IIa, Band 134. Wien.

Fischer, M. (2008): Ungesteuerte und gesteuerte Retention entlang von Fließgewässern - Beurteilung der Wirksamkeit möglicher Maßnahmen unter Verwendung hydrodynamisch-numerischer Modellierung. Berichte des Lehrstuhls und der Versuchsanstalt für Wasserbau und Wasserwirtschaft, TU München, Nr. 119. ISBN 978-3940476-11-1. 2008.

Geilen, N., Jochems, H., Krebs, L., Muller, S. Pedroli, B., Van der Sluis, T., Van Looy, K., Van Rooij, S. (2004): Integration of ecological aspect in flood protection strategies: defining an ecolo- gical minimum. River Research and Applications 20: 269-283.

Gems, B. (2012): Entwicklung eines integrativen Konzeptes zur Modellierung hochwasserrelevanter Prozesse und Bewertung der Wirkung von Hochwasserschutzmaßnahmen in alpinen Talschaften. Modellanwendung auf Basis einer regionalen Betrachtungsebene am Beispiel des Ötztales in den Tiroler Alpen Forum. Innsbruck: innsbruck university press (IUP) (= Forum Umwelttechnik und Wasserbau, 13). ISBN 978-3902811-53-0.

Godina, R., Lalk, P., Lorenz, P., Müller, G., Weilguni, V. (2006): Hochwasser 2005 - Ereignisdokumentation, Teilbericht des Hydrografischen Dienstes. In: Lebensministerium (BMLFUW). Wien.

Habersack, H., Badura, H., Gabriel, H., Hengl, M., Huber, B., Jäger, G.. Knoblauch, H., Krouzecky, N., Lalk, P., Mandl, C., Moser, M., Reindl, R., Rickenmann, D., Sattler, S., Schöberl, F. Tritthart, M. (2011): Fließgewässermodellierung - Feststofftransport und Gewässermorphologie. ÖWAV-Arbeitsbehelf. Wien.

Habersack, H., Wagner, B., Schoder, A., Hauer, C. (2013): Die Bedeutung von Feststoffhaushalt und Sedimentdurchgängigkeit für eine nachhal- 
tige Nutzung der Wasserkraft. Österreichische Wasser- und Abfallwirtschaft 65 (9-10): 354-61. doi:10.1007/s00506-013-0108-0.

Haider, S. (2014b): Retention in unseren Flüssen. Österreichische Wasser- und Abfallwirtschaft 66 (1-2): 59-66.

Haider, S., Walder, S., Federspiel, M. (2014): Hochwasserschutz Tiroler Unterinntal - Kompensation der entfallenden Retention. Internationales Symposium „Wasser- und Flussbau im Alpenraum", VAW-Mitteilungen, ETH Zürich, Nr. 228. Zürich

Haider, S. (2014a): Regionalstudie Unterinntal, Büro Pieler ZT GmbH, Auftraggeber Bundeswasserbauverwaltung Tirol (nicht veröffentlicht). Hofer, B. (1990): Die Geschiebeführung am Unterlauf des Tiroler Inn in Kirchbichl. Österreichische Wasserwirtschaft: 42 , Heft $1 / 2$.

Homagk, P. (2007): Hochwasserschutzkonzept mit gesteuerten und ungesteuerten Retentionsmaßnahmen am Oberrhein. Fachtagung „Flutpolder - Hochwasserrückhaltebecken im Nebenschluss“. Berichte des Lehrstuhls und der Versuchsanstalt für Wasserbau und Wasserwirtschaft, TU München, Nr. 113. ISBN 978-3 940476-03-6. 15-27.

Hunziker, R. P. (1995): Fraktionsweiser Geschiebetransport. Dissertation, VAW-Mitteilungen, ETH Zürich, Nr. 138. Zürich.

Klar, R. (2016): Langzeitsimulation des Geschiebetransports in alpinen Tälern: Weiterentwicklung von Methoden zur Modellierung der langfristigen Sohllagenentwicklung und zur Ermittlung von Hochwassergefahren in inne- ralpinen Tälern. Dissertation, Arbeitsbereich Wasserbau, Universität Innsbruck.

Nujić, M., Hydrotec, Ingenieurgesellschaft für Wasser und Umwelt mbH, Hunziker, Zarn \& Partner AG (2015): Benutzerhandbuch HYDRO FT-2D, Erweiterung zu HYDRO_AS-2D zur Simulation des Stofftransports. Aachen.

ÖWAV (2007): Fließgewässermodellierung Arbeitsbehelf Feststofftransport und Gewässermorphologie. Bundesministerium für Land- und Forstwirtschaft, Umwelt und Wasserwirtschaft. Wien.

Patt, H., Gonsowski, P., Vischer, D. (2011): Wasserbau: Grundlagen, Gestaltung von wasserbaulichen Bauwerken und Anlagen (7. Auflage) Springer-Verlag Berlin Heidelberg, Berlin, Heidelberg.

Phillips, B. C. und Sutherland, A. J. (1989): Spatial lag effects in bed load sediment transport. Journal of Hydraulic Research 27(1): 115-133.

Plörer, M., Achleitner, S., Gems, B., Aufleger, M. (2012): Uncertainty in 2D Flood Plain Modeling Concerning Varying Distribution of Tributary Bed Loads and Rainfall. In: Murillo Muñoz R: River Flow 2012. Proceedings of the International Conference on Fluvial Hydraulics. San José. London: Taylor \& Francis. ISBN 978-0-415-62129-8. 1203-1210.

Poulard, C., Lafont, M., Lenar Matyas, A., Lapuszek, M. (2010): Flood mitigation designs with respect to river ecosystem functions. A problem oriented conceptual approach. Ecological Engineering 36 (1): 69-77.
Rickenmann, D. (1990): Bedload Transport Capacity of Slurry Flows at Steep Slopes. VAW-Mitteilungen, ETH Zürich, Nr. 103. Zürich. Tritthart, M., Liedermann, M., Schober, B., Habersack, H. (2011): Non-uniformity and layering in sediment transport modelling 2: River application. Journal of Hydraulic Research 49(3): 335344. doi:10.1080/00221686.2011.583487. Umach, L. (2013): Bedeutung der Approximation von Kornverteilungskurven bei der zweidimensionalen numerischen Simulation von Geschiebetransportprozessen. Dissertation, Arbeitsbereich Wasserbau, Universität Innsbruck. Wasserrechtsgesetz 1959 (WRG 1959): BGBl. Nr. 215/1959 idgF. BGBl. I Nr. 54/2014. Wien.

Werth, K. (2003): Geschichte der Etsch zwischen Meran und San Michele - Flussregulierung, Trockenlegung der Möser Hochwasser. Tappeiner AG. Lana.

Wiberg, P. L. und Smith, J. D. (1989): Model for Calculating Bed Load Transport of Sediment, Journal of Hydraulic Engineering 115 (1): 101-23. doi:10.1061/(ASCE)0733-9429(1989)115:1(101).

Wong, M., Parker, G. (2006): Reanalysis and Correction of Bed-Load Relation of Meyer-Peter and Müller Using Their Own Database. Journal of Hydraulic Engineering 132 (11): 1159-68.

Zarn, B. (2008): Entwicklungskonzept Alpenrhein. Interpraevent 2008, Dornbirn, Conference Proceedings, Vol. 1: 247-258. 